\title{
Thermodynamics of hydration and oxidation in the proton conductor Gd-doped barium cerate from density functional theory calculations
}

\author{
Jessica Hermet, ${ }^{1,2}$ François Bottin, ${ }^{1}$ Guilhem Dezanneau, ${ }^{2}$ and Gregory Geneste ${ }^{1, *}$ \\ ${ }^{1}$ CEA, DAM, DIF, F-91297 Arpajon, France \\ ${ }^{2}$ Laboratoire Structures, Propriétés et Modélisation des Solides, UMR CNRS 8580, École Centrale Paris, \\ Grande Voie des Vignes, 92295 Châtenay-Malabry Cedex, France \\ (Received 30 March 2012; published 25 May 2012)
}

\begin{abstract}
Hydration and oxidation of gadolinium-doped barium cerate, a system with highly promising properties when used as electrolyte for protonic ceramic fuel cells, are investigated by means of density functional calculations. The energy landscape of oxygen vacancies and interstitial protons in this strongly distorted orthorhombic perovskite is computed. Although the most stable sites for protons are found in the close vicinity of the dopant, the picture of a very complex energy landscape emerges, in which some sites far away from Gd are found more stable than other ones in its close vicinity, due to the highly distorted geometry of the host materials. The fully hydrated phase can be approximated by a structure with 16 local minima. Both hydration (water incorporation) and oxidation (oxygen incorporation) are found to be exothermic processes with reaction enthalpies of $-1.34 \mathrm{eV} / \mathrm{H}_{2} \mathrm{O}$ molecule and $-0.70 \mathrm{eV} / \mathrm{O}$ atom, respectively. The hole polaron resulting from the exothermic incorporation of oxygen is found localized on oxygens around the dopant (small polaron) and carries a spin magnetic moment. Finally, the competition between hydration and oxidation is studied and discussed as a function of oxygen and water partial pressures.
\end{abstract}

DOI: 10.1103/PhysRevB.85.205137

PACS number(s): 66.30.Dn, 71.15.Mb

\section{INTRODUCTION}

Proton conduction phenomena are of crucial importance in a number of technological issues in the electrochemical field, in particular fuel cells, as well as in natural processes, for instance glass and minerals leaching by water, and continue to raise many fundamental questions (diffusion paths, diffusion mechanisms, quantum tunneling of protons). One of the most common proton conductor is water, in which proton conduction takes place through a hop-turn mechanism occurring along a hydrogen-bonded network of $\mathrm{H}_{2} \mathrm{O}$ molecules, and suggested more than two hundred years ago by Grotthuss. ${ }^{1}$ The Grotthuss mechanism is now a general name for the proton hopping/reorientation phenomenon as it occurs in many organic or inorganic compounds. Focusing on materials for fuel cell technology, mainly two kinds of systems involve proton conduction as a central process: In polymer-based electrolytes for fuel cells (proton exchange membrane fuel cells), the protons $\mathrm{H}^{+}$are indeed the charge carriers that constitute the ionic current in the electrolyte, and the low activation energy of the proton hopping allows the fuel cell to work at low temperature. However, another family of fuel cells, with membranes made of ceramics (solid oxides), work at higher temperature. In such devices, ionic conduction in the electrolyte can be ensured by oxygen anions such as in yttrium-doped zirconia, or by protons if the oxide of the electrolyte gets hydrated in presence of vapor, which allows a decrease of the working temperature with respect to anionic conduction. The corresponding devices are called protonic ceramic fuel cells (PCFCs).

This is precisely the case of some perovskite-type oxides that, under aliovalent acceptor doping, might present a significant proton conductivity when exposed to humid conditions. For this reason, such compounds have been proposed since the late 1990s as possible candidates for electrolytes for PCFCs. ${ }^{2}$ In particular, many works have been focused on acceptor-doped perovskites having a tetravalent element on the
B site, such as titanates, zirconates, stannates, and cerates: ${ }^{2-6}$ Substituting the B-site tetravalent cation by a trivalent element globally increases the basicity of the system and, by charge compensation, allows appearance of charged oxygen vacancies $V_{\mathrm{O}}^{\bullet \bullet}$. Such process is usually observed to enhance the reactivity of the system with water: $\mathrm{H}_{2} \mathrm{O}$ molecules can incorporate into the solid and dissociate in the vacancies, giving rise to protonic defects, according to $\mathrm{H}_{2} \mathrm{O}+V_{\mathrm{O}}^{\bullet \bullet}+\mathrm{O}_{\mathrm{O}}^{X} \rightarrow 2 \mathrm{OH}_{\mathrm{O}}^{\bullet}$. These defects usually yield a protonic conductivity, the longrange diffusion of protons resulting from alternative hopping (between two oxygen atoms) and reorientation (around an oxygen atom) motions.

Among the various perovskite compounds exhibiting such properties and candidates as electrolytes for PCFCs, one finds cubic compounds such as $\mathrm{BaZrO}_{3}$ and $\mathrm{BaSnO}_{3}$, and compounds with distorted orthorhombic structures such as alkaline-earth cerates, and especially $\mathrm{BaCeO}_{3}$ (BCO), which exhibits a high protonic conductivity. ${ }^{2-6}$ Both families have been the subject of theoretical investigations ${ }^{7-13}$ but the number of theoretical works dedicated to the latter is much smaller. This is probably due to the high complexity of the orthorhombic structure, which is strongly distorted: Indeed BCO crystallizes in its ground state in a Pnma space group $\left(a^{-} b^{+} a^{-}\right.$in Glazer notation $\left.{ }^{14}\right)$, a structure in which two antiphase octahedra tilting systems (around $x$ and $z$ ) coexist with a third in-phase one (around $y$ ), giving rise to many different inequivalent sites for the proton and for the oxygen vacancy, and thus the possibility of having complex diffusion paths. Indeed, Bilic and Gale ${ }^{15}$ studied the stable sites of protons in orthorhombic In-doped $\mathrm{CaZrO}_{3}$ and the energy barriers separating these sites, showing the high complexity of such a system.

Oxygen octahedra tilts are observed in perovskite compounds in which the B-site cation is rather large with respect to the space left inside the oxygen octahedron. The probability to have a distorted perovskite can be evaluated through the use 
of the well-known Goldschmidt's tolerance factor: ${ }^{16}$

$$
t=\frac{r_{A}+r_{\mathrm{O}}}{\sqrt{2}\left(r_{B}+r_{\mathrm{O}}\right)} .
$$

Considering this tolerance factor, octahedra tilts usually manifest for values of $t$ lower than 1. In BCO, using Shannon ionic radii with appropriate coordination numbers $\left(r_{\mathrm{Ba}}=1.61\right.$ $\left.\AA, r_{\mathrm{Ce}}=0.87 \AA, r_{\mathrm{O}}=1.35 \AA\right),{ }^{17}$ we indeed get a small factor of $t=0.94$, while the tolerance factor for cubic perovskites tends to be slightly above 1 (1.01 for $\mathrm{BaZrO}_{3}$ and 1.03 for $\mathrm{BaSnO}_{3}$ ). Higher values of $t$, by contrast, rather tend to favor polar distortions and the appearance of ferroelectricity (1.06 for $\mathrm{BaTiO}_{3}$ ).

The physicochemical properties of BCO doped with a trivalent element (yttrium and, to a lesser extent, indium) have been theoretically studied, ${ }^{11,12}$ but no first-principles study has been carried out on hydration/protonation and oxidation of Gd-doped BCO, to the best of our knowledge, although substituting $\mathrm{Gd}^{3+}$ to $\mathrm{Ce}^{4+}$ is known to induce a high level of protonic conductivity according to experimental studies. ${ }^{18,19}$ And yet, understanding hydration/protonation and oxidation processes at the atomic scale in cerates could help to improve and design new materials for electrolytes or electrodes in fuel cells. Moreover, $\mathrm{Gd}$ is known to be easily substituted to $\mathrm{Ce}$ in $\mathrm{CeO}_{2}{ }^{20}$ to provide oxygen vacancies and form an anionic conductor widely used as electrolyte in solid oxide fuel cells. Thus, Gd-doped BCO can be viewed as a prototypical system to study hydration and proton conduction in low-symmetry perovskites compounds.

In this work, we present a comprehensive survey of the complex energy landscape of protons and oxygen vacancies in the highly distorted structure of Gd-doped barium cerate (BCGO). The equilibrium geometries of the pure and doped compound are computed and compared to experiments (synthesis of Gd-doped BCO and x-ray diffraction characterization). The stable geometries for the two defects in numerous possible sites are determined by density functional calculations, and the resulting energy landscape is discussed. The hydration reaction, which corresponds to the dissociation of molecular water $\left[\mathrm{H}_{2} \mathrm{O}+V_{\mathrm{O}}^{\bullet \bullet}+\mathrm{O}_{\mathrm{O}}^{X} \rightarrow 2 \mathrm{OH}_{\mathrm{O}}^{\bullet}\right]$ and the oxidation reaction, which corresponds to the incorporation of oxygen $\left[\frac{1}{2} \mathrm{O}_{2}+V_{\mathrm{O}}^{\bullet \bullet} \rightarrow \mathrm{O}_{\mathrm{O}}^{X}+2 h^{\bullet}\right]$, are studied and their enthalpy estimated. They are both found exothermic $(\Delta H<0)$, and the hole polaron resulting from the incorporation of oxygen is characterized. Finally, the thermodynamic conditions of stability of hydrated and oxidized phases of BCGO are determined as a function of oxygen and water chemical potentials, allowing us to discuss how both processes compete with each other.

\section{COMPUTATIONAL DETAILS}

\section{A. Numerical parameters}

We have carried out density functional calculations using the generalized gradient approximation (GGA) in the form proposed by Perdew, Burke, and Ernzerhof ${ }^{21}$ (GGA-PBE), and the projector augmented wave (PAW) method. ${ }^{22,23}$ We have used the ABINIT code ${ }^{24,25}$ together with PAW atomic data that explicitly treat semicore electrons for the metallic elements (Ba: $5 s^{2} 5 p^{6} 6 s^{2}$, Ce: $5 s^{2} 5 p^{6} 4 f^{1} 5 d^{1} 6 s^{2}$, Gd: $5 s^{2} 5 p^{6} 4 f^{7}$ $\left.5 d^{1} 6 s^{2}\right)$. The PAW radii for the atomic data are $2.8(\mathrm{Ba}), 2.5$ $(\mathrm{Ce}$ and $\mathrm{Gd}), 1.4(\mathrm{O})$, and $0.8(\mathrm{H})$ atomic units.

We use a plane-wave cutoff of 20 hartrees and employ an 80-atom supercell ( \pm 1 atom according to the kind of defect we are looking at-dopant, vacancy, or proton). The corresponding Brillouin zone is sampled by a $2 \times 2 \times 2 \mathbf{k}$-point mesh. Because of the presence of magnetic Gd atoms, all the calculations are spin polarized (only collinear magnetism is used; spin-orbit effects are neglected in this study). We have fully optimized the geometry of the system (atomic positions and supercell vectors) using the efficient Broyden-FletcherGoldfarb-Shanno minimization scheme implemented in the ABINIT code, within an optimization criterion on atomic forces of $1.0 \times 10^{-4}$ hartrees/bohr $(\approx 0.005 \mathrm{eV} / \AA)$. Finally, in order to check the good description of the hydroxyl group in these condensed-matter calculations (in particular the short bond length between the oxygen and the proton), a harder atomic data of oxygen (requiring a high plane-wave cutoff of 46 hartrees), with a shorter radius of 1.1 a.u. has also been tested (see Appendix A).

\section{B. Methodology}

In this section, we provide a description of the three families of systems we have simulated in the present work.

\section{Dry compound}

The compound studied in this work is Gd-doped barium cerate (BCGO). This material results from the mixing of the binary oxides $\mathrm{BaO}, \mathrm{CeO}_{2}$, and $\mathrm{Gd}_{2} \mathrm{O}_{3}$, according to

$$
\mathrm{BaO}+(1-\delta) \mathrm{CeO}_{2}+\frac{\delta}{2} \mathrm{Gd}_{2} \mathrm{O}_{3} \rightarrow \mathrm{BaCe}_{1-\delta} \mathrm{Gd}_{\delta} \mathrm{O}_{3-\delta / 2} .
$$

We will thus simulate BCGO as a material with composition $\mathrm{BaCe}_{1-\delta} \mathrm{Gd}_{\delta} \mathrm{O}_{3-\delta / 2}$, denoted hereafter as the "dry" system, in which the $+2,+3$, and +4 oxidation degrees of $\mathrm{Ba}$, $\mathrm{Gd}$, and $\mathrm{Ce}$ are thus preserved. Moreover, it is a common experimental fact ${ }^{20}$ that, even for high values of $\delta$ (up to 0.2), this compound keeps the perovskite structure. Thus it can be viewed as a BCO compound in which some Ce have been substituted by Gd (dopants, with proportion $\delta$ ) and oxygen atoms have been removed (oxygen vacancies, with proportion $\delta / 2$ ). For this reason, BCGO has been simulated by introducing a number of $\mathrm{Gd}$ dopants and $\mathrm{O}$ vacancies in the ratio $2: 1$. We have constructed an 80-atom supercell, which has been first fully optimized without any defect and relaxed in the Pnma space group, which is the ground state structure of BCO. Then two Gd dopants are introduced, as well as one oxygen vacancy, to form BCGO. Formally, this corresponds to $\delta=0.125$. However, it will be shown hereafter (Sec. IV B) that the interaction between defects is rather short-range, and that at such content the dilute limit can be reasonably considered as reached.

Qualitatively, on the basis of the numerical results, it is possible to know which charge state the previous defects, associated inside the supercell, exhibit. Indeed, in the BCGO compound, we find the following:

(i) The $\mathrm{Gd}$ ion is in a +3 ionic form, as shown by the value of its magnetic moment, which is found close to $7 \mu_{B}$ in all the calculations we have performed. As a defect, it therefore 
corresponds formally to a -1 charge state (Kröger-Vink notation $\mathrm{Gd}_{\mathrm{Ce}}^{\prime}$ ).

(ii) The compound is found insulating (this is a requirement for its use as an electrolyte for fuel cells). No electronic defect (electron or hole) is thus present in BCGO.

By considering the rule of charge neutrality and the -1 defect charge on $\mathrm{Gd}$, this means that oxygen vacancies are charged in a +2 state $\left(V_{\mathrm{O}}^{\bullet \bullet}\right)$.

Note that this is exactly what is traditionally assumed in acceptor-doped perovskites, in which the stoichiometry of the initial binary oxides is preserved. The dry compound is presented in detail in Sec. IV A, in which the relative position of the defects has been varied to provide the energy landscape of the oxygen vacancies around dopants.

\section{Hydrated compound}

Second, we also study the hydrated form of BCGO, which results from the incorporation of water according to a chemical reaction which is traditionally written as $\mathrm{H}_{2} \mathrm{O}+V_{\mathrm{O}}^{\bullet \bullet}+\mathrm{O}_{\mathrm{O}}^{X} \rightarrow$ $2 \mathrm{OH}_{\mathrm{O}}^{\bullet}$. This reaction consumes the (charged) oxygen vacancies to produce (twice more) protonic defects. Therefore we obtain the (fully) hydrated compound by introducing in the BCO supercell two Gd dopants and two interstitial hydrogens. These two kinds of defects are in equal number under the assumption of total hydration (a hydrated compound can be thus also obtained by introducing one dopant and one hydrogen).

Here again the charge state of the associated defects can be known, since in the hydrated compound, we find that

(i) Gd is still in a -1 defect charge state, as in BCGO (magnetic moment $\approx 7 \mu_{B}$ );

(ii) the fully hydrated compound is perfectly insulating: No electronic defect (electron or hole) is thus present in the hydrated compound.

There are thus two kinds of defects in the hydrated system: the Gd dopants ( -1 charge state) and the hydrogen interstitials. Therefore the rule of charge neutrality ensures that the hydrogens are in a +1 charge state (protonic defects $\mathrm{OH}_{\mathrm{O}}^{\bullet}$ ), and that charge of the point defects is preserved throughout the hydration reaction. Hydration is presented in detail in Sec. IV B, in which the relative position of the defects has been varied to provide the energy landscape of the protons around dopants.

To summarize, in order to study the dry and hydrated compounds, the defects are systematically placed in the 80 -atom supercell by pairs to provide charge compensation: $\left\{V_{\mathrm{O}}^{\bullet \bullet}+2 \mathrm{Gd}_{\mathrm{Ce}}^{\prime}\right\},\left\{\mathrm{OH}_{\mathrm{O}}^{\bullet}+\mathrm{Gd}_{\mathrm{Ce}}^{\prime}\right\}$, or $\left\{2 \mathrm{OH}_{\mathrm{O}}^{\bullet}+2 \mathrm{Gd}_{\mathrm{Ce}}^{\prime}\right\}$. The first couple of defects ( $\mathrm{O}$ vacancy +2 dopants) corresponds to the system before hydration ("dry"), while the two others (proton + dopant) correspond to the system after (total) hydration.

The charges of the point defects $\left(-1\right.$ for $\mathrm{Gd}_{\mathrm{Ce}}^{\prime}$, +2 for $V_{\mathrm{O}}^{\bullet \bullet}$, +1 for $\mathrm{OH}_{\mathrm{O}}^{\bullet}$ ) are formal values, deduced from stoichiometry considerations related to the oxidation states, as classically done in solid state chemistry and within the Kröger-Vink notation. They are different from the charge that would be obtained from examination of the electron density at the defective site and at the undefective site, by using for instance the Bader method. ${ }^{26}$ In particular, the protonic defect $\mathrm{OH}_{\mathrm{O}}^{\bullet}$ is not a really bare proton and the $\mathrm{O}-\mathrm{H}$ bond keeps an ionocovalent character.

\section{Oxidized compound}

Finally, we have also studied the system after oxygen incorporation into the vacancies (oxidation). This oxidation reaction ${ }^{10}$ is traditionally assumed to occur according to $\frac{1}{2} \mathrm{O}_{2}+V_{\mathrm{O}}^{\bullet \bullet} \rightarrow \mathrm{O}_{\mathrm{O}}^{X}+2 h^{\bullet}$. It consumes vacancies and is supposed to produce electronic defects (holes). The oxidized system is thus studied by introducing in the 80 -atom BCO supercell only the two Gd dopants. Here again the $\mathrm{Gd}$ are found to have $\mathrm{a}+7 \mu_{B}$ magnetic moment, ensuring the +3 oxidation degree of $\mathrm{Gd}$ and the -1 charge state of the defect. But the major difference with the two previous cases is that the system is now found to have a metallic electronic structure, containing empty states at the top of the valence band (holes). Therefore, in this case, charge compensation of the dopant is provided by an electronic defect, namely a hole polaron.

Note that oxygen incorporation could also provide peroxo groups $\mathrm{O}_{2}^{2-}$, as found in Ref. 27 in the $\mathrm{BaSnO}_{3}$ compound. But such species were found unstable and are thus not considered in the present study.

\section{Limitation of the present study}

Finally, the three families of systems studied (dry, hydrated, oxidized) have in common to be various acceptor-doped forms of Gd-doped BCO. In all cases, the electronic chemical potential $\mu_{e}$ (Fermi level) remains close to $\epsilon_{\mathrm{VBM}}$, the top of the valence band. Higher values of $\mu_{e}$ within the band gap would correspond to the presence of donor defects in the system, such as neutral oxygen vacancies, neutral interstitial hydrogens, or electronic polarons. Although such cases might be interesting for the physicochemistry of materials for fuel cells, in particular when these compounds are exposed to reducing atmosphere $\left(\mathrm{H}_{2}\right)$, we have excluded them from the present study.

\section{PRELIMINARY RESULTS: PURE BARIUM CERATE AND GADOLINIUM OXIDE}

The PAW atomic data have been carefully tested on the standard state of each chemical element (bcc Ba, fcc Ce, hcp $\mathrm{Gd}, \mathrm{O}_{2}$ molecule, $\mathrm{H}_{2}$ molecule) and on their corresponding oxidized form $\left(\mathrm{BaO}\right.$ : rocksalt structure, $\mathrm{CeO}_{2}$ : fluorite structure, $\mathrm{Gd}_{2} \mathrm{O}_{3}$ : bixbyite structure, $\mathrm{H}_{2} \mathrm{O}$ molecule). For the solids, we compute the equilibrium volume, the cohesive energy (i.e., with respect to free atoms), and the bulk modulus, and for the molecules the bond lengths and the binding energy. The results are summarized in Table I and compared to experimental data and previous DFT calculations, with which a very good agreement is found.

\section{A. $\mathrm{BaCeO}_{3}$}

Various experimental studies on barium cerate have shown that it undergoes - under ambient pressure - three phase transitions when increasing the temperature. ${ }^{42-44}$ At low temperature, the structure is orthorhombic with space group Pnma $\left(a^{-} b^{+} a^{-}\right.$using Glazer's notations $\left.{ }^{14}\right)$, as already explained. When heating, it first loses the in-phase tilt to turn into the Imma space group $\left(a^{-} b^{0} a^{-}\right)$around $550 \mathrm{~K}$. Then the structure changes through a first-order transition at $670 \mathrm{~K}$ to a rhombohedral phase with space group $R \overline{3} c\left(a^{-} a^{-} a^{-}\right)$. Above 
TABLE I. Atomic (resp. unit cell) volumes for simple metals (resp. for oxides), molecular bond lengths, cohesive or binding energies (with respect to free atoms), and bulk moduli (for solids) from our simulations, compared with experiments and other DFT calculations.

\begin{tabular}{|c|c|c|c|c|}
\hline Element & & $\begin{array}{l}V_{0}\left(\AA^{3} / \text { at. }\right) \\
\text { or } d_{\mathrm{eq}}(\AA)\end{array}$ & $E_{\text {coh }}(\mathrm{eV} /$ f.u. $)$ & $B_{0}(\mathrm{GPa})$ \\
\hline \multirow[t]{4}{*}{$\mathrm{Ba}[\mathrm{bcc}]$} & Present & 63.37 & -1.89 & 8.2 \\
\hline & Exp. $^{28}$ & 63.25 & -1.90 & 10.3 \\
\hline & GGA $^{29}$ & 62.98 & -1.91 & 7.6 \\
\hline & $\mathrm{LDA}^{29}$ & 56.34 & -2.23 & 7.9 \\
\hline \multirow[t]{4}{*}{$\mathrm{Ce}[\mathrm{fcc}]$} & Present & 26.60 & -4.64 & 42.5 \\
\hline & Exp. $^{28}$ & 34.35 & -4.32 & 23.9 \\
\hline & GGA $^{30}$ & 26.05 & & 48.7 \\
\hline & $\mathrm{LDA}^{31}$ & 23.09 & & 59 \\
\hline \multirow{3}{*}{$\begin{array}{l}\text { Gd [hcp] } \\
\text { (FM) }\end{array}$} & Present & 33.45 & -3.95 & 35.9 \\
\hline & Exp. $^{28}$ & 37.89 & -4.14 & 38.3 \\
\hline & $\mathrm{LDA}^{32}$ & 32.60 & & 40.9 \\
\hline \multirow[t]{4}{*}{$\mathrm{O}_{2}$} & Present & 1.229 & -6.26 & \\
\hline & Exp. $^{33}$ & & -5.12 & \\
\hline & $\mathrm{GGA}^{33}$ & & -6.21 & \\
\hline & $\mathrm{LDA}^{22}$ & 1.228 & -7.33 & \\
\hline \multirow[t]{4}{*}{$\mathrm{H}_{2}$} & Present & 0.756 & -4.51 & \\
\hline & Exp. $^{34}$ & 0.741 & -4.75 & \\
\hline & GGA $^{35}$ & 0.750 & -4.54 & \\
\hline & $\mathrm{LDA}^{22}$ & 0.773 & -4.62 & \\
\hline \multirow[t]{3}{*}{$\mathrm{H}_{2} \mathrm{O}$} & Present & 0.976 & -10.14 & \\
\hline & Exp. $^{33}$ & & -10.10 & \\
\hline & GGA & $0.973^{36}$ & $-10.13^{33}$ & \\
\hline \multirow[t]{4}{*}{$\mathrm{BaO}$} & Present & 43.68 & -10.08 & 66.9 \\
\hline & Exp & $42.49^{37}$ & & $66.2^{38}$ \\
\hline & GGA $^{39}$ & 43.41 & & 75.7 \\
\hline & $\mathrm{LDA}^{39}$ & 40.80 & & 91.6 \\
\hline \multirow[t]{4}{*}{$\mathrm{CeO}_{2}$} & Present & 40.97 & -21.32 & 173.5 \\
\hline & $\operatorname{Exp}^{40}$ & 39.61 & & 220 \\
\hline & $\mathrm{GGA}^{41}$ & 41.14 & & 178.0 \\
\hline & $\mathrm{LDA}^{41}$ & 38.93 & & 210.7 \\
\hline
\end{tabular}

$1170 \mathrm{~K}, \mathrm{BaCeO}_{3}$ is finally cubic (no more octahedron tilt) with space group $P m \overline{3} m\left(a^{0} a^{0} a^{0}\right)$.

At the working temperature of a PCFC (usually around $900 \mathrm{~K}$ ), barium cerate is supposed to be in its rhombohedral structure. However, geometry optimizations performed on the $R \overline{3} c$ structure without symmetry show that the material systematically returns in its Pnma structure, showing that $R \overline{3} c$ is not a local minimum in configuration space but is probably stabilized at high temperature by entropy through thermal vibrations. Thus we will only study the materials in its ground state (Pnma space group). The Pnma unit cell and oxygen octahedra tilts are represented in Fig. 1. Due to this tilted structure, there are two nonequivalent positions for the oxygen atoms, usually called apical oxygens (O1) and equatorial oxygens $(\mathrm{O} 2)$. We note that perovskite oxides $A B \mathrm{O}_{3}$ crystallizing in the Pnma space group exhibit three different $B$-O distances: $B-\mathrm{O} 1$ and two different values for $B-\mathrm{O} 2$, that alternate along the $B-\mathrm{O}$ chains in the equatorial plane. In $\mathrm{BaCeO}_{3}$, we have $\mathrm{Ce}-\mathrm{O} 1=2.276 \AA$ while $\mathrm{Ce}-\mathrm{O} 2=2.266 \AA$ and $2.275 \AA$ (see Fig. 3).

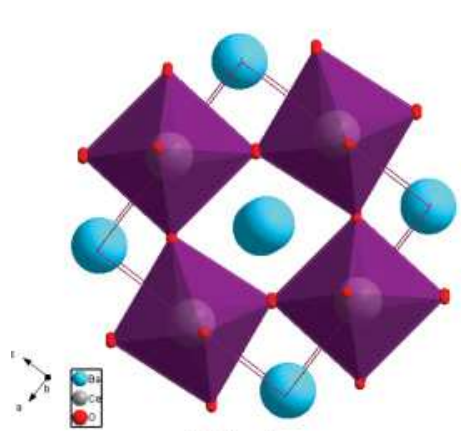

(a) In phase

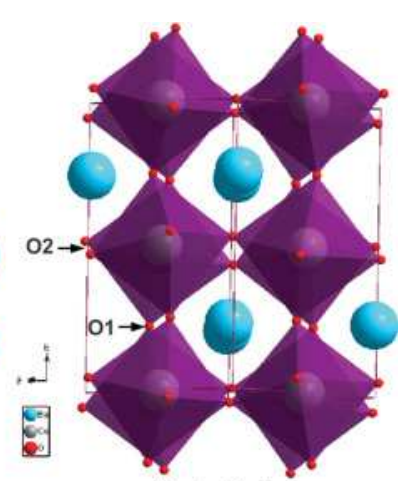

(b) Anti-phase
FIG. 1. (Color online) Structure of $\mathrm{BaCeO}_{3}$ in its Pnma space group with its two tilts: in phase (along $b$ axis) and antiphase (along $a$ and $c$ axis).

Structural and energetic results on $\mathrm{BaCeO}_{3}$-as found from our density functional calculations-are given in Tables II, III, and IV, and compared to other data from experiments or DFT calculations. The unit cell volume, and lattice constants are slightly overestimated (a well-known tendency of GGA), and the formation energy is underestimated by $\approx 9 \%$.

\section{B. Gadolinium sesquioxide $\mathrm{Gd}_{2} \mathrm{O}_{3}$}

Previous $a b$ initio studies on rare-earth sesquioxides ${ }^{50}$ have been mainly focused on the high-temperature hexagonal A-type and monoclinic B-type structures with respectively 5 and 30 atoms in the primitive cell, rather than on the C-type cubic structure, which has a bixbyite primitive cell containing 40 atoms. ${ }^{51}$ The weak number of such studies on $\mathrm{Gd}_{2} \mathrm{O}_{3}$ can be explained not only by the heavy computational cost due to the large number of atoms (and thus electrons), but also by the lack of accurate experimental information on its magnetic structure. Rare-earth compounds are known to frequently exhibit subtle effects due to the presence of strongly correlated electrons in the $4 f$ electronic shell. Since our theoretical approach, based on standard GGA, might fail in the treatment of strongly correlated electrons, we wish to check that it is able to reasonably reproduce the main physicochemical properties of the $\mathrm{Gd}_{2} \mathrm{O}_{3}$ compound (crystal structure, electronic structure, and magnetism). We have thus optimized $\mathrm{Gd}_{2} \mathrm{O}_{3}$ in its ground state bixbyite structure without any special treatment of electronic correlations (such as introducing a Hubbard $U$ parameter).

\section{Bixbyite structure}

At room temperature, gadolinium sesquioxide crystallizes in the bixbyite structure (space group $I a \overline{3}$, body-centered

TABLE II. Equilibrium volume (per primitive cell), formation energy (i.e., with respect to $\mathrm{Ba}, \mathrm{Ce}$, and $\mathrm{O}$ in their standard state), bulk modulus, and band gap of $\mathrm{BaCeO}_{3}$.

\begin{tabular}{lllll}
\hline \hline & $\begin{array}{c}V_{0} \\
\left(\AA^{3} / \text { f.u. }\right)\end{array}$ & $\begin{array}{c}E^{\mathrm{f}} \\
(\mathrm{eV} / \text { f.u. })\end{array}$ & $\begin{array}{c}B_{0} \\
(\mathrm{GPa})\end{array}$ & $\begin{array}{c}\text { Band Gap } \\
(\mathrm{eV})\end{array}$ \\
\hline Present & 350.4 & -15.97 & 107.37 & 2.15 \\
Exp. & $340.8^{45}$ & $-17.52^{46}$ & $103.7^{45}$ & $3.2^{47}$ \\
GGA & $348.58^{48}$ & & & $2.6^{47}$ \\
\hline \hline
\end{tabular}


TABLE III. Lattice constants of pure BCO and BCGO.

\begin{tabular}{|c|c|c|c|c|}
\hline \multirow[b]{2}{*}{ Compound } & & \multicolumn{3}{|c|}{ Cell Parameters $(\AA)$} \\
\hline & & $a$ & $b$ & $c$ \\
\hline \multicolumn{5}{|l|}{$\mathrm{BaCeO}_{3}$} \\
\hline & GGA [Present] & 6.293 & 8.867 & 6.280 \\
\hline & $\mathrm{GGA}^{48}$ & 6.28 & 8.81 & 6.30 \\
\hline & $\mathrm{XRD}^{42}$ & 6.227 & 8.791 & 6.252 \\
\hline \multicolumn{5}{|c|}{$\mathrm{BaCe}_{1-\delta} \mathrm{Gd}_{\delta} \mathrm{O}_{3-\delta / 2}$} \\
\hline \multirow[t]{2}{*}{$\delta=0.125$} & GGA [Present] & 6.287 & 8.889 & 6.287 \\
\hline & XRD [Present] & 6.231 & 8.772 & 6.248 \\
\hline$\delta=0.10$ & $\mathrm{XRD}^{19}$ & 6.221 & 8.770 & 6.244 \\
\hline$\delta=0.15$ & $\mathrm{XRD}^{20}$ & 6.203 & 8.769 & 6.243 \\
\hline$\delta=0.20$ & $\mathrm{XRD}^{20}$ & 6.223 & 8.777 & 6.241 \\
\hline
\end{tabular}

cubic) with a 40-atom primitive cell (see Fig. 2). At higher temperature, it becomes monoclinic $(1425 \mathrm{~K})$ and then hexagonal $(2443 \mathrm{~K}) .{ }^{52}$ In the bixbyite structure, there are two inequivalent sites for $\mathrm{Gd}$ atoms: Four $\mathrm{Gd}^{3+}$ ions are on site $8 b\left(C_{2}\right.$, six identical bonds with oxygen atoms) and $12 \mathrm{Gd}^{3+}$ ions are on site $24 d\left(C_{3 i}\right.$, four short and two longer bonds with oxygen atoms). This bixbyite structure can be seen as a fluorite one where one fourth of the oxygen atoms have been removed. We have performed a full optimization (lattice constant and atomic positions) of $\mathrm{Gd}_{2} \mathrm{O}_{3}$, assuming an antiferromagnetic order for the Gd magnetic moments. Note that the atomic structure is not found to depend on the kind of magnetism, ferromagnetic (FM) or antiferromagnetic (AFM). The results (see Table V) show a good agreement with both experiments and other $a b$ initio calculations for the atomic positions and lattice parameter. The difference between our equilibrium volume $\left(80.67 \AA^{3}\right)$ and the one obtained by Hirosaki et al.$^{50}\left(78.99 \AA^{3}\right)$, also performed in GGA, may be explained by the freezing of the $4 f$ electrons in the core of the pseudopotential used by these authors, whereas we explicitly treat the $4 f$ electrons as valence electrons.

\section{Magnetism}

Whether gadolinium oxide has a FM or AFM ground state is still an open question. Previous density functional calculations on this oxide were mainly dedicated to clusters. However, these calculations show that antiferromagnetic states are in general

TABLE IV. Atomic positions of $\mathrm{BaCeO}_{3}($ Pnma $)$.

\begin{tabular}{lrrrr}
\hline \hline & Present & \multicolumn{1}{c}{ GGA $^{48}$} & \multicolumn{1}{c}{ Exp. $^{49}$} & \multicolumn{1}{c}{ Exp. $^{42}$} \\
\hline $\mathrm{Ba}(x)$ & 0.0254 & 0.0273 & 0.0204 & 0.0123 \\
$\mathrm{Ba}(y)$ & 0.2500 & 0.2500 & 0.2500 & 0.2500 \\
$\mathrm{Ba}(z)$ & -0.0080 & -0.0077 & -0.0085 & -0.0038 \\
$\mathrm{Ce}(x)$ & 0.0000 & 0.0000 & 0.0000 & 0.0000 \\
$\mathrm{Ce}(y)$ & 0.0000 & 0.0000 & 0.0000 & 0.0000 \\
$\mathrm{Ce}(z)$ & 0.5000 & 0.5000 & 0.5000 & 0.5000 \\
$\mathrm{O} 1(x)$ & -0.0199 & -0.0200 & -0.0094 & -0.0089 \\
$\mathrm{O} 1(y)$ & 0.2500 & 0.2500 & 0.2500 & 0.2500 \\
$\mathrm{O} 1(z)$ & 0.4204 & 0.4194 & 0.4287 & 0.4290 \\
$\mathrm{O} 2(x)$ & 0.2802 & 0.2833 & 0.2790 & 0.2707 \\
$\mathrm{O} 2(y)$ & 0.0431 & 0.0446 & 0.0384 & 0.0377 \\
$\mathrm{O} 2(z)$ & 0.7208 & 0.7186 & 0.7235 & 0.7302 \\
\hline \hline
\end{tabular}

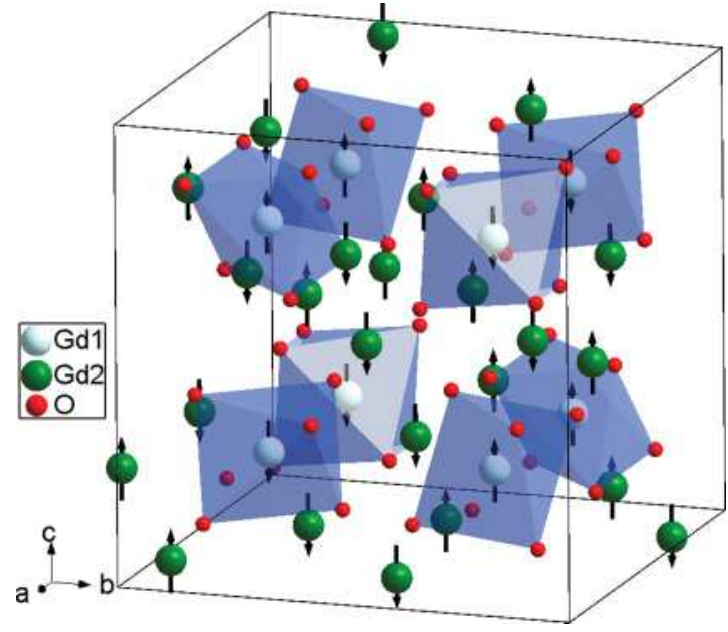

FIG. 2. (Color online) Representation of the bixbyite structure of $\mathrm{Gd}_{2} \mathrm{O}_{3}$ (conventional cubic unit cell-multiplicity $=2$-with 80 atoms) with the AFM order studied in this work.

more stable than the ferromagnetic state ${ }^{55,56}$ except in the case of Perdesen et al ${ }^{57}$ Experimental papers also lean in favor of an antiferromagnetic coupling between nearest-neighbor $\mathrm{Gd}$ ions. ${ }^{54}$

Therefore, we performed two calculations, one on the FM state of $\mathrm{Gd}_{2} \mathrm{O}_{3}$ and one on an AFM state compatible with the space group of the crystal (see Fig. 2): The AFM state is found more stable, though the energy difference between the AFM and FM structures is very small. To complete our study, we also tried a nonmagnetic (NM) structure (with all $f$ bands half filled, to get occupation numbers identical to the FM case), which was found to have a much higher energy than the two magnetic ones. These results are summarized in Table VI and the energy difference between the two ordered magnetic structures is compared with previous results. Our result is in the range with previous values, although the study of clusters instead of bulk may induce a difference on $E_{\mathrm{FM}}-E_{\mathrm{AFM}}$.

\section{Electronic structure}

The electronic density of states (DOS) for both magnetic structures has been computed. We find an insulating structure with a Kohn-Sham band gap of $2.88 \mathrm{eV}$ for the FM state, and $3.27 \mathrm{eV}$ for the AFM state. The gap for the AFM structure appears to be larger than that of the FM one, as Ayuela et al. ${ }^{56}$ and Ning et $a l^{55}$ found in small clusters, using respectively the PBE functional on $\mathrm{Gd}_{2} \mathrm{O}_{3}$ and the B3LYP functional on $\mathrm{Gd}_{12} \mathrm{O}_{18}$. Note that conductivity measurements provide a gap

TABLE V. Structural parameters of $\mathrm{Gd}_{2} \mathrm{O}_{3}$ in its bixbyite structure.

\begin{tabular}{lccccc}
\hline \hline & $u$ & $x$ & $y$ & $z$ & $V\left(\AA^{3}\right)$ \\
\hline $\begin{array}{l}\text { GGA } \\
\text { [Present] }\end{array}$ & -0.0316 & 0.3906 & 0.1507 & 0.3794 & 80.67 \\
GGA $^{50}$ & -0.0319 & 0.3909 & 0.1510 & 0.3793 & 78.99 \\
LSD-SIC $^{53}$ & & & & & 80.70 \\
Expt. $^{54}$ & -0.0304 & 0.3913 & 0.1512 & 0.3811 & 79.02 \\
& $( \pm 0.007)$ & $( \pm 0.0013)$ & $( \pm 0.0012)$ & $( \pm 0.0015)$ & \\
\hline
\end{tabular}


TABLE VI. Energy difference between different magnetic states of $\mathrm{Gd}_{2} \mathrm{O}_{3}$ (in eV by formula unit).

\begin{tabular}{lc}
\hline \hline & $E(\mathrm{FM})-E(\mathrm{AFM})$ \\
\hline Present work & 0.012 \\
$\mathrm{Gd}_{12} \mathrm{O}_{18}$ cluster $^{55}$ & 0.001 \\
$\mathrm{Gd}_{2} \mathrm{O}_{3}$ cluster $^{56}$ & 0.02 \\
\hline \hline
\end{tabular}

of $2.64 \mathrm{eV}^{58}$ whereas optical measurements find a gap up to $5.44 \mathrm{eV}$. $^{59,60}$

Therefore, the use of pure GGA, despite its usual poor description of strongly correlated electrons, is found sufficient to describe with reasonable accuracy the electronic structure of $\mathrm{Gd}_{2} \mathrm{O}_{3}$. In particular, $\mathrm{Gd}_{2} \mathrm{O}_{3}$ is found insulating as in experiments. Table VII summarizes the computed and measured values of the gap, showing that our AFM value is in good agreement with SIC-LSD ${ }^{53}$ (gap for A-type structure) and LDA $+U$ results. ${ }^{61}$

This correct description of a rare-earth oxide, containing strongly correlated electrons, by standard GGA is probably related to the half filling of the $4 f$ shell in the $\mathrm{Gd}^{3+}$ ion, since the electronic configuration $4 f^{7}$ (the 7 electrons having parallel spins) allows the minimization of the electronic repulsion of $f$ electrons (the Pauli principle applies to the orbital parts and the electrons spatially exclude each other by filling the different $4 f$ orbitals) and is strongly stabilized by the exchange effects. We therefore expect a correct description of Gd-doped BCO within the GGA-PBE functional, since Gd conserves the same electronic configuration in this compound.

\section{RESULTS}

We now investigate the chemical processes of hydration and oxidation in BCGO. We first describe the initial state of these reactions (dry system $=$ BCGO), and then turn to its fully hydrated form (which contains protonic defects and no more oxygen vacancy), and finally to its fully oxidized form obtained by incorporation of $\mathrm{O}_{2}$, which dissociates and fills the oxygen vacancies (it provides electronic defects, namely holes, that might destroy the insulating character required to have a good electrolyte).

\section{A. Gd-doped $\mathrm{BaCeO}_{3}$ : The dry compound}

\section{Energy landscape of oxygen vacancies around the dopant}

In the initial, dry system, the charge of the oxygen vacancies $V_{\mathrm{O}}^{\bullet \bullet}$ exactly compensates that of the $\mathrm{Gd}_{\mathrm{Ce}}^{\prime}$ dopants, i.e., one oxygen vacancy for two Gd dopants. Thus we substitute in the 80 -atom supercell two $\mathrm{Ce}$ atoms by two $\mathrm{Gd}$ atoms (they are placed in a relative position that maximizes their distance),

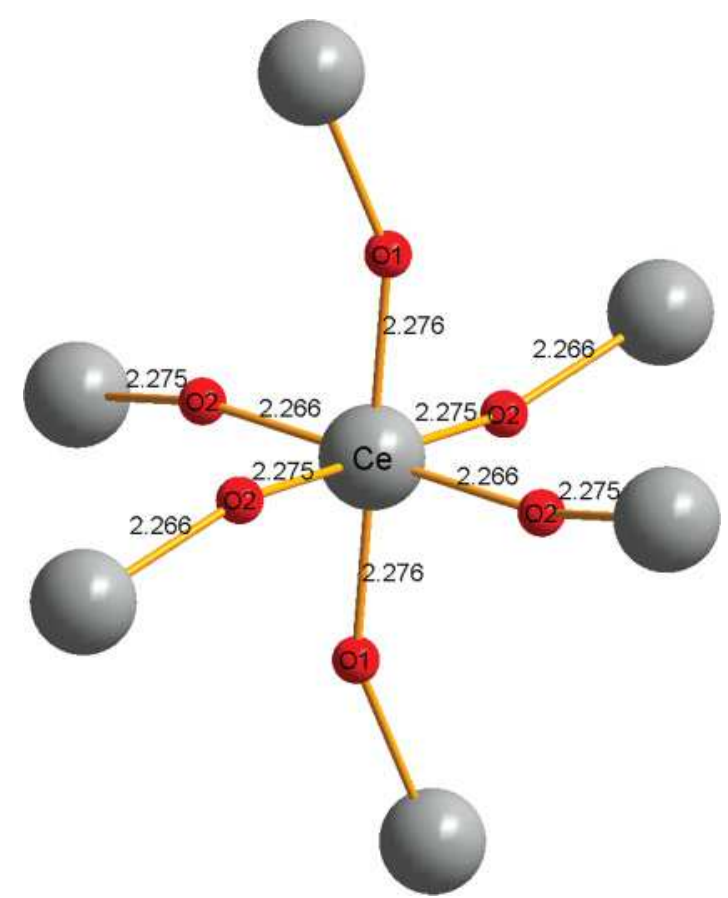

FIG. 3. (Color online) Local environment of the B site in the Pnma structure. There are four equivalent equatorial oxygens $\mathrm{O} 2$, but if the central Ce atom is replaced by a dopant, this equivalence is broken since two $\mathrm{O} 2$ are at $2.266 \AA$ from the $\mathrm{B}$ site and the two others are at $2.275 \AA$ from B (these distances are also modified by the local relaxation subsequent to doping).

and remove one oxygen atom. There are several possibilities to place the vacancy in the neighborhood of a dopant, due to the low symmetry of the structure.

In pure $\mathrm{BCO}$, there would be only two possible positions for the vacancy: $\mathrm{O} 1$ and $\mathrm{O} 2$. However, in the presence of a dopant, this is different: Gd breaks the equivalence between the four equatorial oxygens $(\mathrm{O} 2)$ of its first coordination shell. This is due to the fact that the $B-\mathrm{O}$ distances in the equatorial plane of a perovskite oxide $A B \mathrm{O}_{3}$ in the $P n m a$ structure are of two kinds ( $d_{1}$ and $\left.d_{2}\right)$, which alternate along the $B-\mathrm{O}$ chains (Fig. 3). Thus two of these equatorial oxygens are at $d_{1}$ from $B$ while two others are at $d_{2}$ from $B$. When $B=\mathrm{Gd}$, the set of the four $\mathrm{O} 2$ is therefore split into two sets of two $\mathrm{O} 2$ (called $\mathrm{O} 2$ and $\mathrm{O}_{2}^{\prime}$ in the following), the two oxygens of each set being symmetrically placed with respect to $\mathrm{Gd}$.

We first focus on the vacancy placed on the first coordination shell of $\mathrm{Gd}$ ( 3 possible sites: $\mathrm{O} 1, \mathrm{O} 2$, and $\mathrm{O}_{2}^{\prime}$ ). The calculations show that on this first coordination shell of $\mathrm{Gd}$, the $\mathrm{O} 2$ and $\mathrm{O}_{2}^{\prime}$ sites have very close energies and are thus quasi-identical (energy difference $<0.015 \mathrm{eV}$ ). They are thus considered as one single site in Fig. 4, which shows the

TABLE VII. Experimental and theoretical values of the energy gap of $\mathrm{Gd}_{2} \mathrm{O}_{3}$ in eV for the cubic structure (except in Ref. 53).

\begin{tabular}{|c|c|c|c|c|c|c|c|c|c|c|c|c|}
\hline \multirow{3}{*}{$\begin{array}{l}\text { Ref. } \\
\text { State }\end{array}$} & \multicolumn{4}{|c|}{ Experiments } & \multicolumn{6}{|c|}{ Ab initio Calculations } & \multirow{2}{*}{\multicolumn{2}{|c|}{ Present }} \\
\hline & 58 & 62 & 59 & 63 & 53 & 61 & & & & & & \\
\hline & & & & & & & FM & $\mathrm{AFM}$ & FM & AFM & FM & AFM \\
\hline Gap (eV) & 2.64 & 5.3 & 5.44 & 3.60 & 3.13 & 3.9 & 2.03 & 2.25 & 4.03 & 4.19 & 2.88 & 3.27 \\
\hline
\end{tabular}




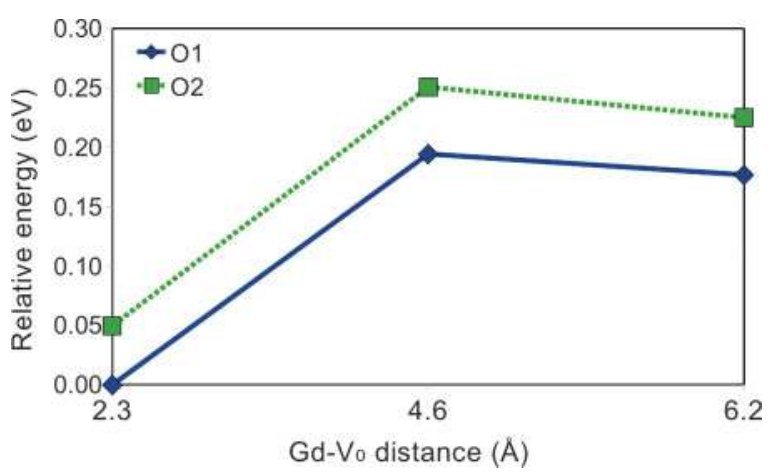

FIG. 4. (Color online) Energies of the different positions for the oxygen vacancy $(\mathrm{eV})$, relative to the most stable one $(\mathrm{O} 1$ in the oxygen octahedron surrounding the dopant). The difference $\mathrm{O} 2 / \mathrm{O} 2^{\prime}$ is ignored even in the first coordination shell due to the energetic proximity of the corresponding configurations.

energy for the different configurations as a function of the Gd-vacancy distance. Beyond the first coordination shell of $\mathrm{Gd}$, the symmetry-breaking produced by the dopant is more complicated. Nevertheless, since the difference $\mathrm{O} 2 / \mathrm{O}^{\prime}$ is insignificant near $\mathrm{Gd}$, we can consider that, beyond the first coordination sphere, there are only two inequivalent oxygen sites: $\mathrm{O} 1$ and $\mathrm{O} 2$ (the dopant is considered as far enough so as to recover the equivalence between all $\mathrm{O} 2$ ). Two $\mathrm{O} 1$ and two $\mathrm{O} 2$ sites are examined in that case, with increasing Gd-vacancy distance up to $\approx 6 \AA$.

We find that the dopant and the vacancy, which are defects having opposite charge, are attracted to each other since the most stable positions for the $\mathrm{O}$ vacancy are in the first coordination shell of the dopant (see Fig. 4). More precisely, the most stable site is the $\mathrm{O} 1$ site in this first coordination shell. The dopant-vacancy interaction is found rather short-range, as the positions beyond the first coordination shell have very close total energies.

From this observation, we can define an approximate interaction energy (sometimes called "association energy") as the mean value (on $\mathrm{O} 1$ and $\mathrm{O} 2$ sites) of the energy difference between the closest and the farthest positions, which gives $E_{\text {int }}\left(\mathrm{Gd}_{\mathrm{Ce}}^{\prime}-V_{\mathrm{O}}^{\bullet \bullet}\right) \approx-0.18 \mathrm{eV}$.

\section{Comparison with experiments}

To compare with experiments, we focus on the most stable system theoretically obtained, i.e., corresponding to the $\mathrm{O}$ vacancy in the $\mathrm{O} 1$ position close to a dopant, and retain its lattice parameters after full optimization (Table III). Of course, the Pnma symmetry is lost due to the presence of the defects, but the octahedron tilting system remains of the type $a^{-} b^{+} a^{-}$, showing that on average, despite the defects, a Pnma space group is preserved in BCGO.

From the experimental point of view, under aliovalent doping, especially at high dopant concentration, perovskite oxides can have their space group modified, or at least have their lattice parameters significantly changed. However, in the case of $\mathrm{BaCeO}_{3}$, the substitution of $\mathrm{Ce}^{4+}$ by trivalent ions is known to have little influence on the structure, even if a shift in the phase transition temperatures ${ }^{64,65}$ is usually observed. This suggests that BCGO keeps the Pnma structure at zero and room temperature.
Experimental data on BCGO at a level of doping of $12.5 \%$ are unfortunately lacking in the literature. Therefore, in order to compare the simulations with experiments, we coupled this theoretical study to an experimental work, and performed $\mathrm{x}$-ray diffraction experiments on BCGO containing exactly $12.5 \%$ of $\mathrm{Gd}$. The compound has been synthesized using the freeze-drying method. After dissolving barium, cerium, and gadolinium acetate powders in water in the stoichiometric ratio, this solution was homogenized and sprayed into liquid nitrogen to get frozen droplets. The solvent was then eliminated by sublimation and the resulting nanopowder was pre-calcined at $600^{\circ}$ for $10 \mathrm{~min}$. Finally, the grayish powder was calcined at $1200^{\circ}$ for $4 \mathrm{~h}$. X-ray diffraction was then performed to check whether there were impurity phases or not and to determine the cell parameter through Rietveld refinement. The parameters found (Table III) are almost identical to the ones of previous experiments on $\mathrm{BaCeO}_{3}$ doped with $10 \%$ of gadolinium.

Both calculations and experiments (Table III) show that there is no significant difference in the lattice parameters between pure $\mathrm{BCO}$ and $\mathrm{BCGO}$ even at this quite large doping $(12.5 \%)$.

\section{B. The hydrated compound and the hydration enthalpy}

Hydration is the process by which the dry compound incorporates water molecules, which dissociate in the vacancies, providing protonic defects (hydroxyl groups) $(\mathrm{OH})_{\mathrm{O}}^{\bullet}$. It is described by the reaction

$$
\mathrm{H}_{2} \mathrm{O}+V_{\mathrm{O}}^{\bullet \bullet}+\mathrm{O}_{\mathrm{O}}^{X} \rightarrow+2(\mathrm{OH})_{\mathrm{O}}^{\bullet} .
$$

\section{Energy landscape of protonic defects}

In the 80-atom supercell, one Ce is substituted by one $\mathrm{Gd}$, and one hydrogen interstitial is introduced, assuming total hydration (complete disappearance of oxygen vacancies). Hydrogen interstitials bind to the oxygen atoms, forming hydroxyl groups $\mathrm{OH}_{\mathrm{O}}^{\circ}$ that roughly point along the [100]-type directions (in perovskites with large lattice parameter) in the interoctahedral space (4 sites per oxygen atom). However, due to the low symmetry of the Pnma space group, there are many possible unequivalent positions for the proton, which we have to rationalize. More precisely, as in the case of the oxygen vacancy, the (equatorial) oxygens $\mathrm{O} 2$ in the first coordination shell of $\mathrm{Gd}$ are of two types, $\mathrm{O} 2$ and $\mathrm{O} 2^{\prime}$, due to the alternating distances along the $B$-O chains of the perovskite structure.

First we focus on protons bonded to the oxygen atoms first neighbor of the Gd dopant, for which the $\mathrm{O} 2 / \mathrm{O}^{\prime}$ dissymmetry leads to the study of many sites: As there are three kinds of oxygen atoms in this shell $\left(\mathrm{O} 1, \mathrm{O} 2\right.$, and $\left.\mathrm{O} 2^{\prime}\right)$, and four possible sites for the proton on each oxygen atom, there are 12 possible positions in the first coordination shell of Gd. Almost no difference is found between the four sites of $\mathrm{O} 2$ (denoted $2 a, 2 b, 2 c$, and $2 d)$ and the corresponding sites of $\mathrm{O}^{\prime}\left(2 a^{\prime}\right.$, $\left.2 b^{\prime}, 2 c^{\prime}, 2 d^{\prime}\right)$, whereas the four sites of O1 $(1 a, 1 b, 1 c, 1 d)$ have very different energies (see Table VIII). Therefore we can consider reasonably that the dissymmetry introduced by the Gd dopant can be neglected, and the protonic sites of $\mathrm{O} 2$ and $\mathrm{O}^{\prime}$ identified. The 8 relevant sites are indicated in Fig. 5. Among these various sites, the $1 a$ is found as the most stable. 
TABLE VIII. Energies (in eV) of the protonic sites in the first coordination shell of Gd, relative to the most stable one $(1 a)$.

\begin{tabular}{lllc}
\hline \hline Site & $\mathrm{O} 1$ & $\mathrm{O} 2$ & $\mathrm{O} 2^{\prime}$ \\
\hline$a$ & $(1 a) 0.000$ & $(2 a) 0.165$ & $\left(2 a^{\prime}\right) 0.163$ \\
$b$ & $(1 b) 0.008$ & $(2 b) 0.052$ & $\left(2 b^{\prime}\right) 0.052$ \\
$c$ & $(1 c) 0.112$ & $(2 c) 0.147$ & $\left(2 c^{\prime}\right) 0.134$ \\
$d$ & $(1 d) 0.003$ & $(2 d) 0.085$ & $\left(2 d^{\prime}\right) 0.088$ \\
\hline \hline
\end{tabular}

Then we examine the protonic sites beyond the first coordination shell of $\mathrm{Gd}$. Since the $\mathrm{O} 2 / \mathrm{O} 2^{\prime}$ dissymmetry has been found negligible near $\mathrm{Gd}$, it will not be considered for oxygen atoms that do not belong to the first coordination shell of Gd. The 8 sites shown in Fig. 5 near Gd are thus examined on oxygen sites beyond. We choose these oxygen sites as belonging to the neighborhood of $\mathrm{Ce}$ atoms that we denote by $\mathrm{Ce} 1, \mathrm{Ce} 2$, and $\mathrm{Ce} 3$ by increasing the $\mathrm{Gd}-\mathrm{Ce}$ distance (see Fig. 5). The energies of all these configurations are shown in Fig. 6, together with those of the sites of the first coordination shell of $\mathrm{Gd}$ (with no distinction between $\mathrm{O} 2$ and $\mathrm{O}^{\prime}$, as explained).

Examination of these energies shows that

(i) the energy landscape of the proton around a Ce site is globally preserved, whatever the site (provided the oxygen site does not also belong to the first coordination shell of a Gd);

(ii) $\mathrm{Ce} 1, \mathrm{Ce} 2$, and $\mathrm{Ce} 3$ exhibit very close energy landscapes, suggesting that protons do not interact any more with the dopant as soon as they leave the first coordination shell of Gd (convergence in the proton-dopant interaction energy is reached);

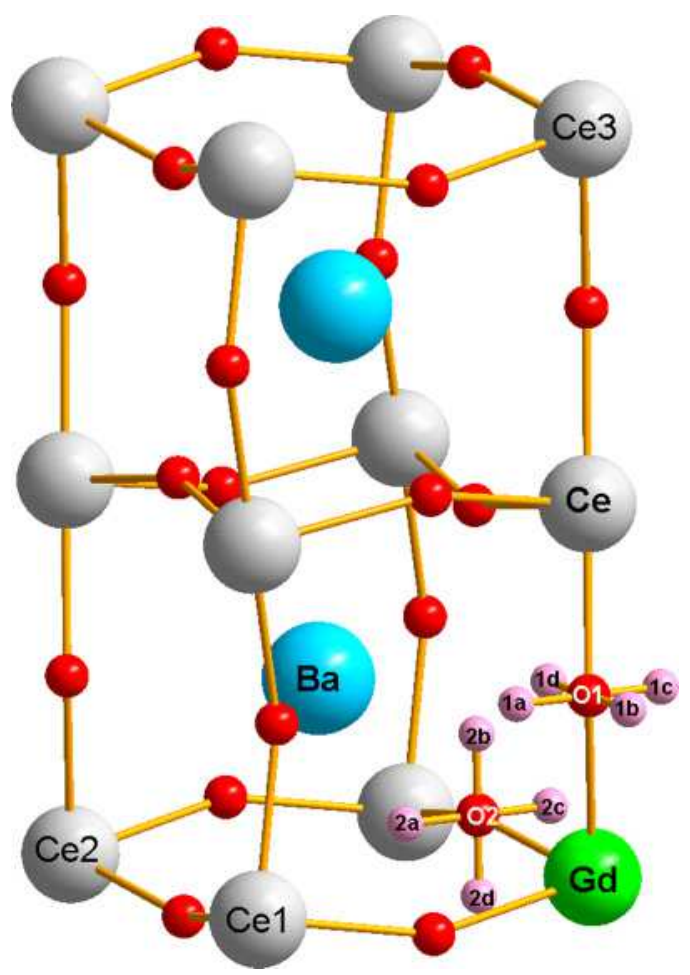

FIG. 5. (Color online) The eight possible positions for a proton bonded to an oxygen atom first neighbor of a dopant.

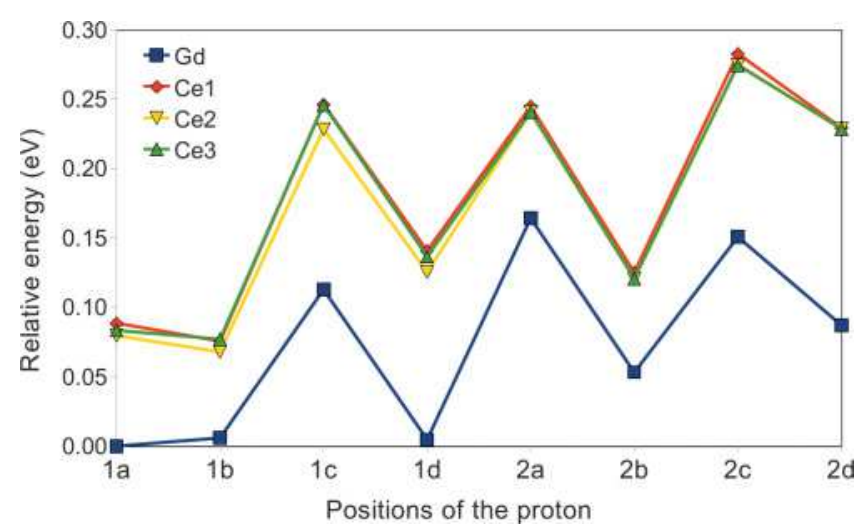

FIG. 6. (Color online) Energies of the 8 possible positions for the proton near different $\mathrm{B}$ sites, relative to the most stable one (i.e., $1 a$ position near $\mathrm{Gd})$.

(iii) as in the vacancy case, the proton is, on average, the most stable when it is in the first-neighbor shell of a $\mathrm{Gd}$ atom because of the opposite charges of the two defects $(-1$ and +1 , respectively);

(iv) however, some positions far from the dopant are more stable than other ones close to $\mathrm{Gd}$, such as $1 b$ of $\mathrm{Ce} 1, \mathrm{Ce} 2$, or $\mathrm{Ce} 3$, which is more stable than $1 c, 2 a$, and $2 c$ near the dopant. Thus the picture that emerges from these calculations is that of a complex energy landscape, different from the traditional picture in which sites close to the dopant act as traps for protons. $^{66}$

We can thus distinguish only two families of sites: when protons are bonded to an oxygen first neighbor of a dopant (8 positions) or not (8 positions). Therefore, we conclude that the protonic energy landscape in $\mathrm{Gd}$-doped $\mathrm{BaCeO}_{3}$ can be reasonably approximated by a structure consisting of 16 local minima, with relative energies shown on Fig. 6.

As we did with the vacancy, we define an approximate dopant-proton interaction energy ("association energy") by averaging (over the possible positions) the energy difference between the first set of values (around $\mathrm{Gd}$ ) and the set corresponding to the furthest site $(\mathrm{Ce} 3)$, which provides $-0.11 \mathrm{eV}$.

\section{Hydration enthalpy}

In order to determine the hydration enthalpy, i.e., the enthalpy of the reaction $\mathrm{H}_{2} \mathrm{O}+\mathrm{V}_{\mathrm{O}}^{\bullet \bullet}+\mathrm{O}_{\mathrm{O}}^{X} \rightarrow+2(\mathrm{OH})_{\mathrm{O}}^{\bullet}$, we perform a calculation with the same doping rate for the initial state and the final state: We thus recompute the energy of the supercell with two protons and two dopants (at the same positions as for the calculation of the vacancy). Both protons are in their lowest energy position, i.e., position $1 a$, close to each dopant. For the vacancy, we also take the most stable state, which is the $\mathrm{O}_{1}$ first neighbor of a dopant. The hydration enthalpy $\Delta H_{\text {hyd }}$ is estimated from the total energies associated with each term of Eq. (1):

$$
\begin{aligned}
\Delta H_{\mathrm{hyd}}= & E_{\mathrm{tot}}(\mathrm{BCO}+2 \mathrm{Gd}+2 \mathrm{H}) \\
& -E_{\mathrm{tot}}\left(\mathrm{BCO}+2 \mathrm{Gd}+V_{\mathrm{O}}\right)-E_{\mathrm{tot}}\left(\mathrm{H}_{2} \mathrm{O}\right),
\end{aligned}
$$

in which $E_{\mathrm{tot}}(\mathrm{BCO}+2 \mathrm{Gd}+2 \mathrm{H})$ is the total energy of the (fully relaxed) supercell containing 2 dopants and 2 protons, $E_{\text {tot }}\left(\mathrm{BCO}+2 \mathrm{Gd}+V_{\mathrm{O}}\right)$ is the total energy of the (fully 
TABLE IX. Present theoretical results compared to experimental hydration enthalpies of acceptor-doped $\mathrm{BaCeO}_{3}$, with various dopants $(\mathrm{Gd}, \mathrm{Y})$. Values measured in Y-doped $\mathrm{BaZrO}_{3}$ and $\mathrm{Y}$-doped $\mathrm{BaSnO}_{3}$ are shown for comparison.

\begin{tabular}{lcclc}
\hline \hline Work & B site & Dopant & Rate $(\%)$ & $\Delta H_{\text {hyd }}(\mathrm{eV})$ \\
\hline Present & Ce & Gd & 12.5 & -1.34 \\
Ref. 3 & Ce & Gd & 15 & -1.78 \\
Ref. 2 & Ce & Y & 10 & -1.68 \\
& Sn & Y & 50 & -0.92 \\
& Zr & Y & 10 & -0.78 \\
\hline \hline
\end{tabular}

relaxed) supercell containing 2 dopants and one $\mathrm{O}$ vacancy, and $E_{\text {tot }}\left(\mathrm{H}_{2} \mathrm{O}\right)$ is the total energy of an isolated water molecule. Since (i) the systems are fully relaxed (atomic positions and stresses) and (ii) the defects are in their most stable positions, the computed quantity $\Delta H_{\text {hyd }}$ is the hydration enthalpy at zero pressure and zero temperature.

The calculations provide a hydration enthalpy of $-1.34 \mathrm{eV} / \mathrm{H}_{2} \mathrm{O}$ molecule, showing that hydration of $\mathrm{Gd}$-doped BCO is an exothermic process, favored at low temperature. This high value is consistent with experimental studies on acceptor-doped $\mathrm{BaCeO}_{3}$ as shown in Table IX. Note that even if we consider less stable positions for the proton or the vacancy, we still obtain an absolute value for the hydration enthalpy above $1 \mathrm{eV}$.

\section{The oxidized compound and the oxidation enthalpy}

Since BCGO contains charged oxygen vacancies, the question may be asked of its possibility to incorporate oxygen. ${ }^{67}$ Although such process cannot result in an effective oxidation of the metallic elements present in the system (which are already in their maximal oxidation state), oxygen incorporation might provide appearance of electronic defects, namely holes, likely to affect the electrical properties of the system. This process, if possible, would occur, in contact with an oxygen atmosphere, according to the oxidation reaction: $\frac{1}{2} \mathrm{O}_{2}+V_{\mathrm{O}}^{\bullet \bullet} \rightarrow \mathrm{O}_{\mathrm{O}}^{X}+2 h^{\bullet}$.

In the final state (BCGO without vacancy), the charge compensation of point defects is no longer fulfilled: Instead of having a vacancy $V_{\mathrm{O}}^{\bullet \bullet}$ for two dopants $\mathrm{Gd}_{\mathrm{Ce}}^{\prime}$, there are only two dopants in the supercell, providing two holes. In a real device, this phenomenon should be avoided as much as possible to preserve the insulating character of the electrolyte. The associated oxidation enthalpy $\Delta H_{\mathrm{ox}}$ has been estimated by computing a new configuration with neither vacancies nor proton, but only two dopants (this configuration is here again fully relaxed).

\section{Exothermic character of oxidation}

As we did for hydration, we consider the two dopants as far as possible in the supercell. The oxidation enthalpy is obtained by $\Delta H_{\mathrm{ox}}=E_{\mathrm{tot}}(\mathrm{BCO}+2 \mathrm{Gd})-E_{\mathrm{tot}}(\mathrm{BCO}+2 \mathrm{Gd}+$ $\left.V_{\mathrm{O}}\right)-\frac{1}{2} E_{\text {tot }}\left(\mathrm{O}_{2}\right)$, where $E_{\text {tot }}(\mathrm{BCO}+2 \mathrm{Gd})$ is the total energy of the (fully relaxed) supercell containing $2 \mathrm{Gd}$ (and no vacancy), and $E_{\text {tot }}\left(\mathrm{O}_{2}\right)$ is the total energy of an isolated $\mathrm{O}_{2}$ molecule. Since (i) the systems are fully relaxed (atomic positions and stresses) and (ii) the defects are in their most stable positions, the computed quantity $\Delta H_{\mathrm{ox}}$ is the oxidation enthalpy at zero pressure and zero temperature. We obtain $\Delta H_{\mathrm{ox}}=-0.70 \mathrm{eV} / \mathrm{O}$ atom. The process is thus exothermic (as hydration) and should be favored at low temperatures (except if kinetically blocked), and unfavored at high temperatures, since it is obviously associated to a $\Delta S<0$.

In several other acceptor-doped perovskites, such as barium zirconate ${ }^{8,10}$ and barium stannate, ${ }^{10}$ this oxidation process has already also been found to be exothermic by DFT calculations, and associated with the appearance of hole polarons and a metallic character.

From the experimental point of view, hole conduction under oxygen atmosphere is indeed observed in these systems, but at high temperature only (proportional to $p_{\mathrm{O} 2}^{1 / 4}$, provided the major defect remains the oxygen vacancies and that hole concentration $\left.[h] \ll\left[V_{\mathrm{O}}\right]\right)$. It is not observed at low temperature. We suggest that the reason is a kinetic blocking: To incorporate into the perovskite network, the $\mathrm{O}_{2}$ molecule (i) should dissociate at the surface and (ii) its progression throughout the materials should occur via the diffusion of oxygen vacancies. Among these two processes, at least the latter is associated to a high activation energy barrier (typically $\approx 0.8 \mathrm{eV}$ ). Note that the hydration, by contrast, is usually associated in acceptor-doped perovskites to smaller energy barriers of $\approx 0.4-0.5 \mathrm{eV}$ (for proton diffusion), and should not be kinetically blocked except at very low temperature.

Moreover, as discussed in Ref. 10, an exothermic process can be made compatible with a hole conductivity increasing with temperature at high temperature provided the hole mobility, which is $\propto e^{-E_{a} / k_{B} T}$ (in the hypothesis of conductivity mediated by small polarons), increases faster than the hole concentration decreases. The latter being $\propto$ $p_{\mathrm{O} 2}^{1 / 4} e^{-\Delta H_{\mathrm{ox}} / 2 k_{B} T}\left(\Delta H_{\mathrm{ox}}\right.$ is the oxidation enthalpy per oxygen atom and is thus $<0$ ), it means that the hole conductivity is $\propto e^{-\left[E_{a}+(1 / 2) \Delta H_{\mathrm{ox}}\right] / k_{B} T}$ and can thus be an increasing function of temperature, provided $E_{a}+\frac{1}{2} \Delta H_{\mathrm{ox}}>0$, that is, if $E_{a}>$ $\frac{1}{2}\left|\Delta H_{\mathrm{ox}}\right|$ (since $\Delta H_{\mathrm{ox}}<0$ ). In the present case, we have $\frac{1}{2}\left|\Delta H_{\mathrm{ox}}\right|=0.35 \mathrm{eV}$ from the DFT calculation. This should therefore imply $E_{a}>0.35 \mathrm{eV}$ for the energy barrier of the hole polaron.

\section{Characteristics of the hole polaron}

Figure 7 represents the spin-up and spin-down electronic density of states (DOS) of the oxidized system. Unoccupied states with well-defined spin polarization appear at the top of the valence band (these empty states do not exist in the insulating dry reference system), reflecting the metallic character of the oxidized system and the creation of holes subsequent to oxygen incorporation. It is possible to characterize the holes by looking at the spatial density of probability of the corresponding unoccupied states. Otherwise Fig. 8 shows the projection of the DOS on the $p$ orbitals of oxygen and the $f$ orbitals of $\mathrm{Gd}$. It clearly indicates that the hole is mainly localized on the oxygen atoms first neighbors of $\mathrm{Gd}$ and supports the picture of a small polaron, for which the mobility is therefore thermally activated and $\propto e^{-E_{a} / k_{B} T}$. Moreover, the magnetic moment of the whole system after incorporation of one $\mathrm{O}$ atom differs by $2 \mu_{B}$, meaning that the holes have the same spin. The small polaron therefore constitutes a magnetic 


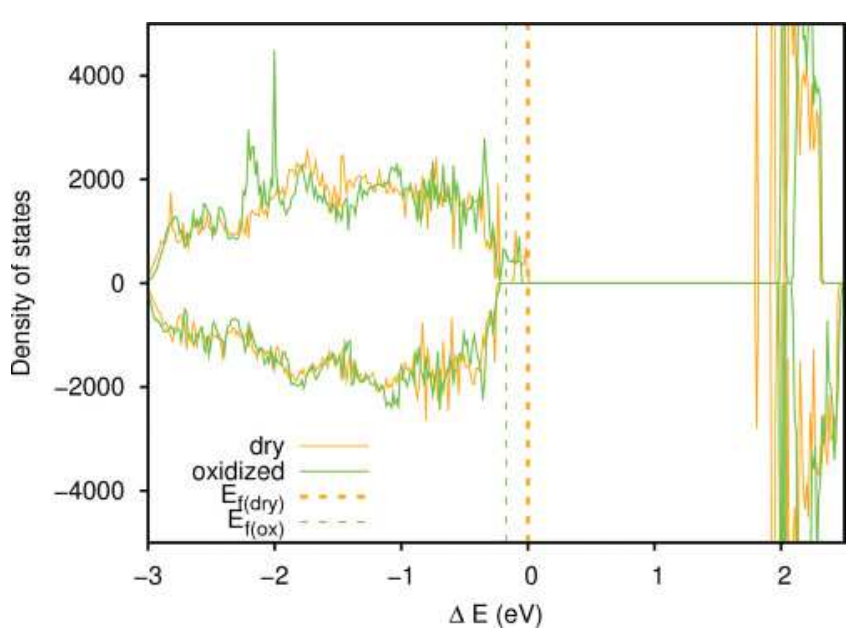

FIG. 7. (Color online) Density of state of the dry (orange line) and oxidized (green line) compounds.

defect (the same magnetization has been found in oxidized barium stannate and barium zirconate ${ }^{10}$ ).

\section{THERMODYNAMICS OF HYDRATION AND OXIDATION IN BCGO}

The previous section was dedicated to the study of hydration and oxidation in BCGO from a microscopic point of view. The hydration and oxidation enthalpies have been determined, as well as the complex energy landscape of protons and vacancies in the compound. However, such data do not provide information about the thermodynamics of hydration and oxidation, i.e., at finite temperature and in various chemical environments, nor about their competition in the simultaneous presence of oxygen and water. ${ }^{68-70}$ From the point of view of thermochemistry, this is because the standard entropy of hydration and oxidation cannot be simply evaluated. In this section, the thermodynamics of hydration and oxidation in BCGO is treated. The stability of the BCGO compound itself with respect to its chemical elements and their oxides is treated in Appendix B.

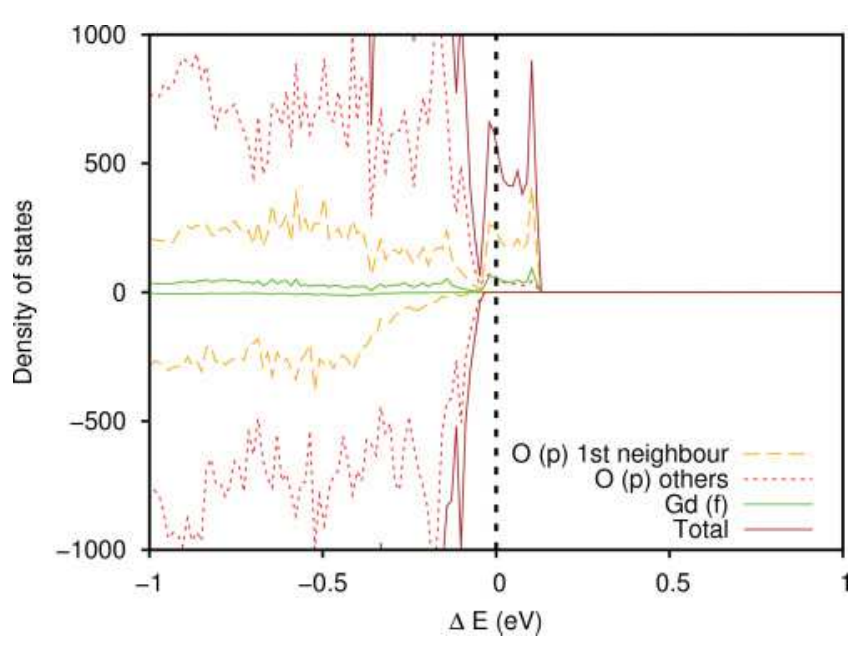

FIG. 8. (Color online) Projected density of state on $p$ orbitals of oxygen and $f$ orbitals of gadolinium.
To address this question, we define the three following phases,

(1) dry (dry) or $\mathrm{BaCe}_{1-\delta} \mathrm{Gd}_{\delta} \mathrm{O}_{3-\delta / 2}$,

(2) oxidized (oxi) or $\mathrm{BaCe}_{1-\delta} \mathrm{Gd}_{\delta} \mathrm{O}_{3}$,

(3) hydrated (hyd) or $\mathrm{BaCe}_{1-\delta} \mathrm{Gd}_{\delta} \mathrm{O}_{3} \mathrm{H}_{\delta}$,

and proceed by calculating their grand potential, defined in a general way by $\Omega=E-T S-\sum_{j} N_{j} \mu_{j}$, where $E$ is the internal energy, and $N_{j}$ and $\mu_{j}$ are respectively the number of atoms and the chemical potential of the $j$ chemical species. We also define the deviation of chemical potential of species $j$ as $\Delta \mu_{j}=\mu_{j}-E_{j}$, and the formation energy of a phase as $E^{f}=E-\sum_{j} E_{j}, E_{j}$ being the internal energy of the $j$ species in its standard phase.

Using the calculated formation energies given in Table XI, one easily gets the three grand potentials as a function of $\Delta \mu_{\mathrm{O}}$ and $\Delta \mu_{\mathrm{H}_{2} \mathrm{O}}$ :

$$
\begin{gathered}
\Omega_{\mathrm{dry}}=E_{\mathrm{dry}}^{f}-c+\frac{\delta}{2} \Delta \mu_{\mathrm{O}}, \\
\Omega_{\mathrm{oxi}}=E_{\mathrm{oxi}}^{f}-c, \\
\Omega_{\mathrm{hyd}}=E_{\mathrm{hyd}}^{f}-c-\frac{\delta}{2}\left(E_{\mathrm{H}_{2} \mathrm{O}}^{f}+\Delta \mu_{\mathrm{H}_{2} \mathrm{O}}-\Delta \mu_{\mathrm{O}}\right),
\end{gathered}
$$

where $c$ is a function of $\Delta \mu_{\mathrm{Ba}}, \Delta \mu_{\mathrm{Ce}}, \Delta \mu_{\mathrm{Gd}}$, and $\Delta \mu_{\mathrm{O}}$, identical for each of the three compounds: $c=\Delta \mu_{\mathrm{Ba}}+$ $(1-\delta) \Delta \mu_{\mathrm{Ce}}+\delta \Delta \mu_{\mathrm{Gd}}+3 \Delta \mu_{\mathrm{O}}$. The expression of $\Omega_{\mathrm{hyd}}$ is obtained from $\Omega_{\text {hyd }}=E_{\text {hyd }}^{f}-c-\delta \Delta \mu_{\mathrm{H}}$, where we have replaced $\Delta \mu_{\mathrm{H}}$ in order to get the dependency in $\Delta \mu_{\mathrm{H}_{2} \mathrm{O}}$, using $\Delta \mu_{\mathrm{H}_{2} \mathrm{O}}=\Delta \mu_{\mathrm{O}}+2 \Delta \mu_{\mathrm{H}}-E_{\mathrm{H}_{2} \mathrm{O}}^{f}$.

Thus, it is possible to plot stability domains for the three phases as a function of $\left(\Delta \mu_{\mathrm{H}_{2} \mathrm{O}}, \Delta \mu_{\mathrm{O}}\right)$, i.e., as a function of the external conditions, since the chemical potentials can be directly related to the partial pressures in the two gases $P_{\mathrm{O}_{2}}$ and $P_{\mathrm{H}_{2} \mathrm{O}}$, and to the temperature. For an ideal gas, $\Delta \mu_{\mathrm{O}}=\frac{1}{2}\left[\mu_{\mathrm{O}_{2}}\left(T, P^{0}\right)+k_{B} T \ln \left(P_{\mathrm{O}_{2}} / P^{0}\right)\right], P^{0}$ being the reference pressure of 1 bar and $\mu_{\mathrm{O}_{2}}\left(T, P^{0}\right)$ taken from experimental values. ${ }^{71}$ The stability zones are plotted in Fig. 9.

Figure 9 illustrates the competition between hydration and oxidation. It shows that the presence of water pushes at high $P_{\mathrm{O}_{2}}$ the oxidation (diagonal line), i.e., extends the electrolyte-like behavior compared to the oxidized state. This

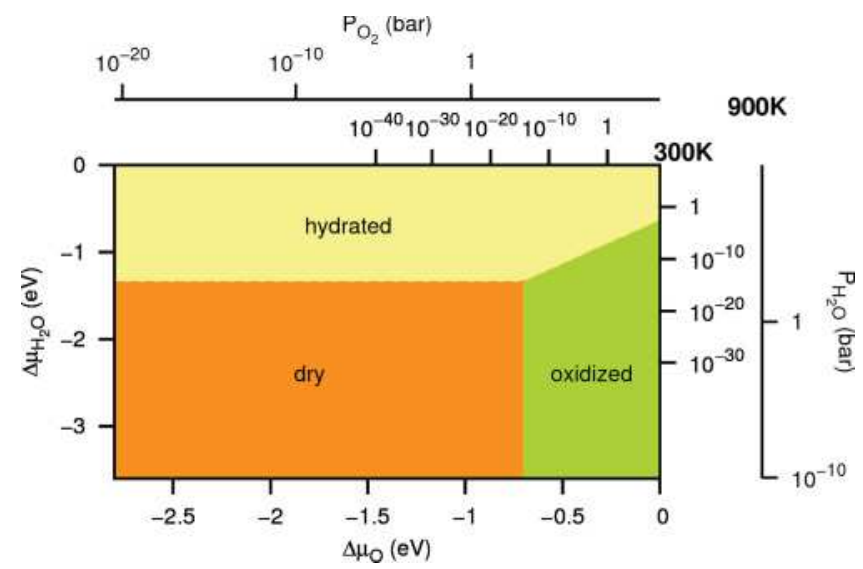

FIG. 9. (Color online) Stability of BCGO in its three possible phases (dry, hydrated, oxidized), as a function of $\left(\Delta \mu_{\mathrm{H}_{2} \mathrm{O}}, \Delta \mu_{\mathrm{O}}\right) . \Delta \mu_{i}$ can be related either to the partial pressure $P_{i}$ at fixed temperature (which is done above) or to the temperature $T$ at fixed partial pressure. 
explains why the oxidized phase is probably absent in the operating conditions of fuel cells. At $T=300 \mathrm{~K}$, if we maintain a water pressure around 25 mbar or above, the compound should be hydrated rather than oxidized, which is in qualitative agreement with experiments (predominance of protons at low $T$ while holes are usually dominant at high $T$ ).

At this stage, we point out that this stability diagram neglects the configurational and vibrational entropy of the BCGO in the various phases (due to the multiple possible sites for $\mathrm{O}$ vacancies and protons), although its impact is probably important. Moreover, we only consider here three phases (fully oxidized, fully hydrated, and dry): intermediate phases with partial incorporation of water or oxygen is expected to lead to blurry regions where the compound is partially hydrated and/or oxidized. The limit between two phases may then be spread over a wide range of water and oxygen partial pressures, instead of being the neat line drawn on Fig. 9. However, the present approach, even if approximate, shows that, at room temperature, the BCGO compound can be hydrated.

\section{CONCLUSION}

In this paper, we have presented a study of the thermodynamics of Gd-doped barium cerate in relation with important physicochemical processes involved in fuel cells, in which this compound can be used as an electrolyte. More precisely, we have characterized by density-functional calculations the hydration and oxidation of the compound.

Under hydration (which is found strongly exothermic), the protons evolve in a very complex energy landscape that can be approximated with a very good accuracy by a structure with 16 local minima ( 8 different protonic sites on the oxygens belonging to the first coordination shell of Gd dopants, 8 sites on other oxygens). In particular, some sites far away from the dopants are more stable than other ones close to them, even though Gd dopants remain, on average, attractive for the protonic defects (association energy $\approx-0.1 \mathrm{eV}$ ). This result questions the traditional picture ${ }^{66}$ according to which dopants, which are formally negative point defects, attract the protons and act as traps for protons. This peculiar feature is the consequence of the highly distorted tilted structure of the host compound.

The modelization of fully hydrated BCGO by a structure with 16 local minima provides a common picture for future kinetic Monte Carlo simulations of proton conduction.

Oxidation, i.e., oxygen incorporation, is also found exothermic, as already found in other acceptor-doped perovskites. ${ }^{10}$ This process is associated with the metallization of the system with appearance of magnetic hole polarons localized on the oxygen atom first neighbors of the Gd dopants.

The present approach applies to the study of hydration and oxidation in any acceptor-doped perovskites or more complex oxides such as mixed ionic-electronic conductors. The chemical behavior of such systems under reducing atmosphere, i.e., in the presence of hydrogen $\mathrm{H}_{2}$, could also be investigated in future works.

\section{ACKNOWLEDGMENTS}

This work was partially performed using HPC resources from GENCI-CCRT/CINES (Grants No. 2010-096468 and No. 2011-096468).
TABLE X. Formation enthalpy in $\mathrm{eV}$ of some compounds for two different calculations

\begin{tabular}{lcc}
\hline \hline & $\begin{array}{c}\mathrm{rc}=1.1 \text { bohrs } \\
(\mathrm{PW} \text { cutoff }=46 \text { hartrees })\end{array}$ & $\begin{array}{c}\mathrm{rc}=1.4 \text { bohrs } \\
(\mathrm{PW} \text { cutoff }=20 \text { hartrees })\end{array}$ \\
\hline $\mathrm{O}_{2}$ & -6.20 & -6.26 \\
$\mathrm{BaO}$ & -5.00 & -5.06 \\
$\mathrm{CeO}_{2}$ & -10.31 & -10.42 \\
$\mathrm{Gd}_{2} \mathrm{O}_{3}$ & -17.21 & -17.39 \\
$\mathrm{BaCeO}_{3}$ & -15.80 & -15.97 \\
dry & -15.47 & -15.64 \\
oxidized & -15.52 & -15.69 \\
hydrated & -15.71 & -15.88 \\
\hline \hline
\end{tabular}

\section{APPENDIX A: COMPARISON WITH A "HARD" PSEUDOPOTENTIAL FOR OXYGEN}

Complementary calculations have been done using a pseudopotential for oxygen with a PAW radius much shorter (1.1 atomic units) than the one used above (1.4 atomic units). This PAW atomic data is supposed to be very accurate but requires a much higher plane wave (PW) cutoff (46 hartrees) to get results with the same accuracy. We have checked that it provides quasi-identical results as the one used above. Some results for formation enthalpies are shown in Table X. It appears that the "soft" pseudopotential tends to slightly overestimate formation energies by $1 \%$. This difference seems rather acceptable considering the huge gain in computational cost, especially if we are computing difference of formation energies (as we did for thermodynamic stability): For instance the difference of formation enthalpy between $\mathrm{BaCeO}_{3}$ and $\mathrm{BaO}+\mathrm{CeO}_{2}$ is the same $(0.49 \mathrm{eV})$ whatever the pseudopotential. We can finally notice that the difference between formation enthalpies computed with soft and hard pseudopotential is exactly the same $(0.17 \mathrm{eV})$ for pure $\mathrm{BaCeO}_{3}$ and all the three phases of Gd-doped $\mathrm{BaCeO}_{3}$.

\section{APPENDIX B: STABILITY OF THE BCGO COMPOUND}

The stability of the BCGO compound is examined, independently of its ability to hydrate or oxidize; i.e., we try to determine the conditions in which BCGO is more stable than the pure elements ( $\mathrm{Ba}, \mathrm{Ce}, \mathrm{Gd})$ in their metallic state, and their oxides. We proceed as above, by computing the grand potential $\Omega=E^{f}-\sum_{j} N_{j} \Delta \mu_{j}$ as a function of the deviations of chemical potentials of each element.

For instance, the grand potential of pure barium cerate is computed according to

$$
\Omega=E_{\mathrm{BaCeO}_{3}}^{f}-\Delta \mu_{\mathrm{Ba}}-\Delta \mu_{\mathrm{Ce}}-3 \Delta \mu_{\mathrm{O}},
$$

with

$$
E_{\mathrm{BaCeO}_{3}}^{f}=E_{\mathrm{BaCeO}_{3}(\text { bulk })}-E_{\mathrm{Ba}(\mathrm{s})}-E_{\mathrm{Ce}(\mathrm{s})}-\frac{3}{2} E_{\mathrm{O}_{2}(\mathrm{~g})} .
$$


Determining the stability of BCGO consists in finding the zone in the space $\left(\Delta \mu_{\mathrm{O}}, \Delta \mu_{\mathrm{Ce}}, \Delta \mu_{\mathrm{Ba}}, \Delta \mu_{\mathrm{Gd}}\right)$ where the grand potential of $\mathrm{BaCe}_{1-\delta} \mathrm{Gd}_{\delta} \mathrm{O}_{3-\delta / 2}$ is lower than that of the elementary metals, gases, and their related oxides.

One of the variables, for instance $\Delta \mu_{\mathrm{Ba}}$, can be eliminated by using

$$
E_{\mathrm{BCGO}}^{f}=\Delta \mu_{\mathrm{Ba}}+(1-\delta) \Delta \mu_{\mathrm{Ce}}+\delta \Delta \mu_{\mathrm{Gd}}+\left(3-\frac{\delta}{2}\right) \Delta \mu_{\mathrm{O}} .
$$

To prevent the formation of metals, gases, and binary oxides, the chemical potential of $\mathrm{Ba}, \mathrm{Ce}, \mathrm{Gd}$, and $\mathrm{O}$ must be smaller in $\mathrm{BaCe}_{1-\delta} \mathrm{Gd}_{\delta} \mathrm{O}_{3-\delta / 2}$ than in other phases:

$$
\begin{gathered}
\Delta \mu_{\mathrm{O}}<0, \Delta \mu_{\mathrm{Ce}}<0, \Delta \mu_{\mathrm{Gd}}<0, \Delta \mu_{\mathrm{Ba}}<0, \\
\Delta \mu_{\mathrm{Ba}}+\Delta \mu_{\mathrm{O}}<E_{\mathrm{BaO}}^{f}, \\
\Delta \mu_{\mathrm{Ce}}+2 \Delta \mu_{\mathrm{O}}<E_{\mathrm{CeO}_{2}}^{f}, \\
\Delta \mu_{\mathrm{Gd}}+3 \Delta \mu_{\mathrm{O}}<E_{\mathrm{Gd}_{2} \mathrm{O}_{3}}^{f} .
\end{gathered}
$$

We finally get the four following conditions from equations (B1)-(B5):

(1) No precipitation of Ba:

$\delta \Delta \mu_{\mathrm{Gd}} \geqslant E_{\mathrm{BCGO}}^{f}-(1-\delta) \Delta \mu_{\mathrm{Ce}}-\left(3-\frac{\delta}{2}\right) \Delta \mu_{\mathrm{O}}$.

(2) No precipitation of $\mathrm{BaO}$ :

$\delta \Delta \mu_{\mathrm{Gd}} \geqslant E_{\mathrm{BCGO}}^{f}-E_{\mathrm{BaO}}^{f}-(1-\delta) \Delta \mu_{\mathrm{Ce}}-\left(2-\frac{\delta}{2}\right) \Delta \mu_{\mathrm{O}}$.

(3) No precipitation of $\mathrm{CeO}_{2}$ :

$$
\Delta \mu_{\mathrm{Ce}} \leqslant E_{\mathrm{CeO}_{2}}^{f}-2 \Delta \mu_{\mathrm{O}}
$$

(4) No precipitation of $\mathrm{Gd}_{2} \mathrm{O}_{3}$ :

$$
\Delta \mu_{\mathrm{Gd}} \leqslant \frac{E_{\mathrm{Gd}_{2} \mathrm{O}_{3}}^{f}}{2}-\frac{3}{2} \Delta \mu_{\mathrm{O}}
$$

As the results cannot be easily represented on a 3D diagram, we plot the precipitation lines of all binary oxides and pure elements in the plane $\Delta \mu_{\mathrm{Gd}}=-5 \mathrm{eV}$ using the enthalpy values of Table XI (the doping rate $\delta$ is equal to $12.5 \%$ ). If

TABLE XI. Formation enthalpies of binary oxides, of BCO and $\operatorname{BCGO}(\delta=0.125)$.

\begin{tabular}{lcr}
\hline \hline & \multicolumn{2}{c}{ Formation Enthalpy (eV/f.u.) } \\
\cline { 2 - 3 } & GGA [our work] & Exp. \\
\hline $\mathrm{H}_{2} \mathrm{O}$ & -2.50 & $-5.78^{72}$ \\
$\mathrm{BaO}$ & -5.06 & $-11.30^{73}$ \\
$\mathrm{CeO}_{2}$ & -10.42 & $-18.58^{52}$ \\
$\mathrm{Ce}_{2} \mathrm{O}_{3}$ & & $-18.94^{52}$ \\
$\mathrm{Gd}_{2} \mathrm{O}_{3}$ & -17.39 & $-17.52^{46}$ \\
$\mathrm{BaCeO}_{3}$ & -15.97 & $-17.18^{74}$ \\
dry & -15.64 & \\
oxidized & -15.69 & \\
hydrated & -15.88 & \\
\hline \hline
\end{tabular}
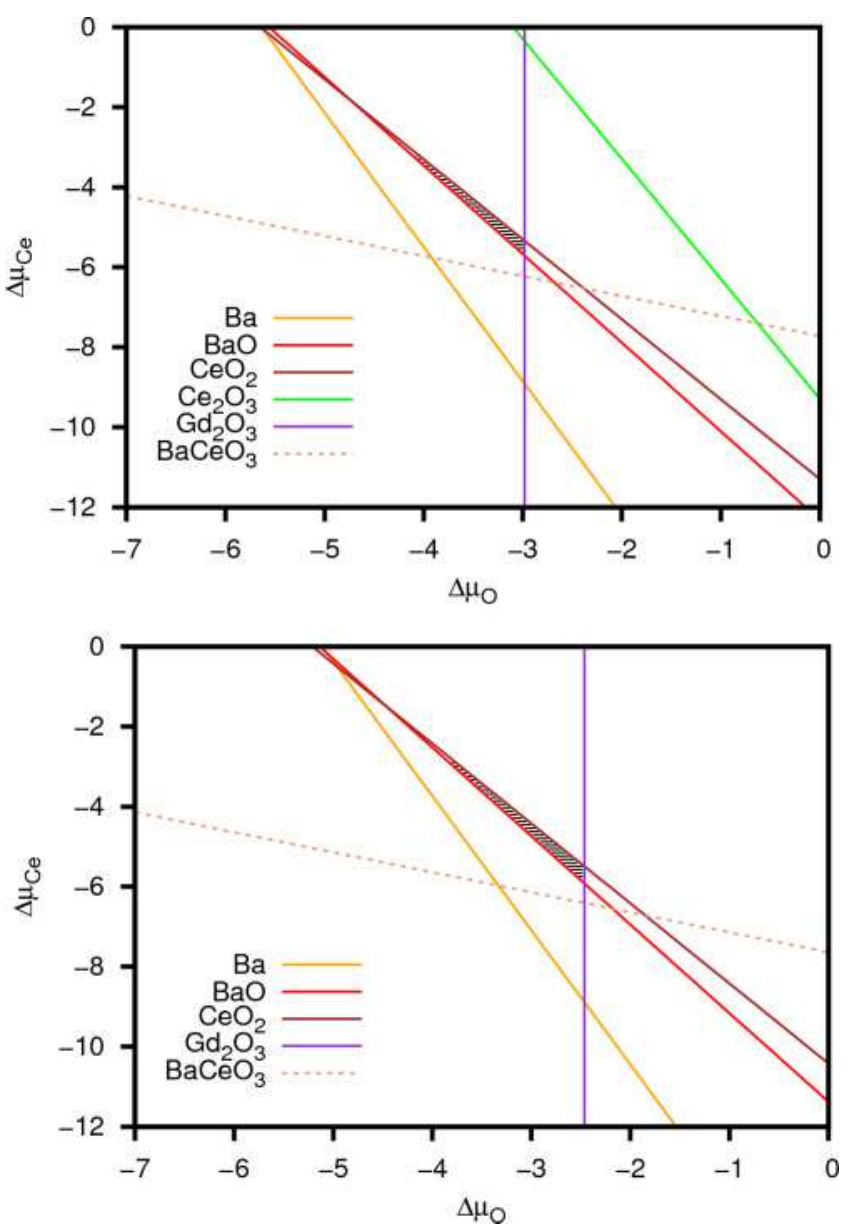

FIG. 10. (Color online) Thermodynamics stability diagram as a function of $\mathrm{O}$ and Ce chemical potentials, in the plane $\Delta \mu_{\mathrm{Gd}}=-5 \mathrm{eV}$. Hatched area corresponds to the stable zone of BCGO (without considering the precipitation of $\mathrm{BaCeO}_{3}$ ).

we choose another $\Delta \mu_{\mathrm{Gd}}=c$ plane, we get the same kind of diagram, but shifted to lower values of $\Delta \mu_{\mathrm{O}}$, and higher values of $\Delta \mu_{\mathrm{Ce}}$ as we increase $\Delta \mu_{\mathrm{Gd}}$ [considering inequality (B9), one can easily see that increasing $\Delta \mu_{\mathrm{Gd}}$ requires decreasing $\left.\Delta \mu_{\mathrm{O}}\right]$.

As a comparison, we also plot the "experimental" diagram, using the same equations (B6)-(B9), but using experimental formation enthalpies taken from the literature, and adding the precipitation line of $\mathrm{Ce}_{2} \mathrm{O}_{3}$. From the precipitation lines, we deduce the stability zone of BCGO, represented by the hatched area in Fig. 10. If we now add the condition of the nonformation of $\mathrm{BaCeO}_{3}$, we find that BCGO is not stable whatever the values of chemical potentials [the supplementary inequality combined with (B7) and (B9) leads to a contradiction].

This surprising unstability of BCGO with respect to $\mathrm{BaCeO}_{3}$ and $\mathrm{Gd}_{2} \mathrm{O}_{3}$, which we obtain even by using the experimental values of formation energies, is in apparent contradiction with experiments, since BCGO has been synthesized in a stable form (in the present work and in previous ones). This can be explained by kinetics: Indeed, BCGO with Gd and $\mathrm{Ce}$ disordered on the B sublattice is probably stable in the high-temperature conditions in which the compound is elaborated, due to the high configurational entropy associated 
with the disorder on the B site. At low temperature, this stability might be lost (order-disorder transition as in binary mixtures) and the system should separate into a $\mathrm{BaCeO}_{3}$ phase and a $\mathrm{Gd}_{2} \mathrm{O}_{3}$ phase. However, it is probable that the
B-sublattice configuration is frozen and kinetically blocked in a high-temperature pattern since the diffusion of $\mathrm{Ce} / \mathrm{Gd}$ atoms throughout the B sublattice at low temperature should involve complex mechanisms with very high energy barriers. *gregory.geneste@cea.fr

${ }^{1}$ C. J. T. V. Grotthuss, Ann. Chem. 58, 54 (1806).

${ }^{2}$ K. D. Kreuer, Solid State Ionics 125, 285 (1999).

${ }^{3}$ K. Kreuer, Solid State Ionics 97, 1 (1997).

${ }^{4}$ K. D. Kreuer, T. Dippel, Y. Baikov, and J. Maier, Solid State Ionics 86-88, 613 (1996).

${ }^{5}$ T. Schober, Solid State Ionics 109, 1 (1998).

${ }^{6}$ P. Murugaraj, K. D. Kreuer, T. He, T. Schober, and J. Maier, Solid State Ionics 98, 1 (1997).

${ }^{7}$ M. E. Björketun, P. G. Sundell, and G. Wahnström, Faraday Discuss. 134, 247 (2007).

${ }^{8}$ P. G. Sundell, M. E. Björketun, and G. Wahnström, Phys. Rev. B 73, 104112 (2006).

${ }^{9}$ É. Bévillon and G. Geneste, Phys. Rev. B 77, 184113 (2008).

${ }^{10}$ E. Bévillon, G. Dezanneau, and G. Geneste, Phys. Rev. B 83, 174101 (2011).

${ }^{11}$ A. Cammarata, A. Martorana, and D. Duca, J. Phys. Chem. A 113, 6381 (2009).

${ }^{12}$ A. Cammarata, A. Emanuele, A. Martorana, and D. Duca, J. Phys. Chem. A 115, 1676 (2011).

${ }^{13}$ M. A. Gomez, M. A. Griffin, S. Jindal, K. D. Rule, and V. R. Cooper, J. Chem. Phys. 123, 094703 (2005).

${ }^{14}$ A. M. Glazer, Acta Crystallogr. Sect. B 28, 3384 (1972).

${ }^{15}$ A. Bilic and J. D. Gale, Chem. Mater. 19, 2842 (2007).

${ }^{16}$ V. M. Goldschmidt, Skrifter Norske Vidensk. Akad. Oslo 1 Mat.Nat. Kl. 2, 1 (1926).

${ }^{17}$ R. D. Shannon, Acta Crystallogr. Sect. A 32, 751 (1976).

${ }^{18}$ T. He, K. D. Kreuer, Y. M. Baikov, and J. Maier, Solid State Ionics 95, 301 (1997).

${ }^{19}$ E. Gorbova, V. Maragou, D. Medvedev, A. Demin, and P. Tsiakaras, Solid State Ionics 179, 887 (2008).

${ }^{20}$ M. Khandelwal, A. Venkatasubramanian, T. R. S. Prasanna, and P. Gopalan, J. Eur. Ceram. Soc. 31, 559 (2011).

${ }^{21}$ J. P. Perdew, K. Burke, and M. Ernzerhof, Phys. Rev. Lett. 77, 3865 (1996).

${ }^{22}$ P. E. Blöchl, Phys. Rev. B 50, 17953 (1994).

${ }^{23}$ M. Torrent, F. Jollet, F. Bottin, G. Zérah, and X. Gonze, Comput. Mater. Sci. 42, 337 (2008).

${ }^{24}$ X. Gonze, B. Amadon, P.-M. Anglade, J.-M. Beuken, F. Bottin, P. Boulanger, F. Bruneval, D. Caliste, R. Caracas, M. Côté, et al. , Comput. Phys. Commun. 180, 2582 (2009).

${ }^{25}$ F. Bottin, S. Leroux, A. Knyazev, and G. Zérah, Comput. Mater. Sci. 42, 329 (2008).

${ }^{26}$ R. W. Bader, Chem. Rev. 91, 893 (1991).

${ }^{27}$ Y. Wang, E. Bévillon, A. Chesnaud, G. Geneste, and G. Dezanneau, J. Phys. Chem. C 113, 20486 (2009).

${ }^{28}$ C. Kittel, Introduction to Solid State Physics (John Wiley \& Sons, 1996), pp. 57-59, 7th ed.

${ }^{29}$ D. Belger, Z. Hüsges, E. Voloshina, and B. Paulus, J. Phys.: Condens. Matter 22, 275504 (2010).

${ }^{30}$ P. Söderlind, O. Eriksson, B. Johansson, and J. M. Wills, Phys. Rev. B 50, 7291 (1994).
${ }^{31}$ B. Amadon, F. Jollet, and M. Torrent, Phys. Rev. B 77, 155104 (2008).

${ }^{32}$ M. Heinemann and W. M. Temmerman, Phys. Rev. B 49, 4348 (1994).

${ }^{33}$ J. Paier, R. Hirschl, M. Marsman, and G. Kresse, J. Chem. Phys. 122, 234102 (2005).

${ }^{34}$ K. P. Huber and G. Hertzberg, Molecular Spectra and Molecular Structure IV: Constants of Diatomic Molecules (Van Norstrand Reinhold, New York, 1979).

${ }^{35}$ D. E. Jiang and E. A. Carter, Phys. Rev. B 70, 064102 (2004).

${ }^{36}$ J. Yang, S. Meng, L. Xu, and E. G. Wang, Phys. Rev. B 71, 035413 (2005).

${ }^{37}$ L. Liu and W. A. Bassett, J. Geophys. Res. 77, 4934 (1972).

${ }^{38}$ S. T. Weir, Y. K. Vohra, and A. L. Ruoff, Phys. Rev. B 33, 4221 (1986).

${ }^{39}$ R. G. Amorim, M. Veríssimo-Alves, and J. P. Rino, Comput. Mater. Sci. 37, 349 (2006).

${ }^{40}$ L. Gerward, J. S. Olsen, L. Petit, G. Vaitheeswaran, V. Kanchana, and A. Svane, J. Alloys Compd. 400, 56 (2005).

${ }^{41}$ S. Fabris, S. de Gironcoli, S. Baroni, G. Vicario, and G. Balducci, Phys. Rev. B 71, 041102 (2005).

${ }^{42}$ K. Knight, Solid State Ionics 145, 275 (2001).

${ }^{43}$ A. V. Kuzmin, V. P. Gorelov, B. T. Melekh, M. Glerup, and F. W. Poulsen, Solid State Ionics 162-163, 13 (2003).

${ }^{44}$ Z. Zhang, J. Koppensteiner, W. Schranz, J. B. Betts, A. Migliori, and M. A. Carpenter, Phys. Rev. B 82, 014113 (2010).

${ }^{45}$ J. Zhang, Y. Zhao, H. Xu, B. Li, D. J. Weidner, and A. Navrotsky, Appl. Phys. Lett. 90, 161903 (2007).

${ }^{46}$ E. H. P. Cordfunke, A. S. Booij, and M. E. Huntelaar, J. Chem. Thermodyn. 30, 437 (1998).

${ }^{47}$ Y. Yuan, J. Zheng, X. Zhang, Z. Li, T. Yu, J. Ye, and Z. Zou, Solid State Ionics 178, 1711 (2008).

${ }^{48}$ J. Bennett and A. Rappe, APS Meeting Abstracts, 2008 (unpublished).

${ }^{49}$ J. Li, U. G. Singh, J. W. Bennett, K. Page, J. C. Weaver, J.-P. Zhang, T. Proffen, A. M. Rappe, S. Scott, and R. Seshadri, Chem. Mater. 19, 1418 (2007).

${ }^{50}$ N. Hirosaki, S. Ogata, and C. Kocer, J. Alloys Compd. 351, 31 (2003).

${ }^{51}$ B. Wu, M. Zinkevich, F. Aldinger, D. Wen, and L. Chen, J. Solid State Chem. 180, 3280 (2007).

${ }^{52}$ M. Zinkevich, Prog. Mater. Sci. 52, 597 (2007).

${ }^{53}$ L. Petit, A. Svane, Z. Szotek, and W. M. Temmerman, Phys. Rev. B 72, 205118 (2005).

${ }^{54}$ R. M. Moon and W. C. Koehler, Phys. Rev. B 11, 1609 (1975).

${ }^{55}$ L. Ning, Y. Zhang, Z. Cui, M. I. Trioni, and G. P. Brivio, J. Phys. Chem. A 112, 13650 (2008).

${ }^{56}$ A. Ayuela, N. H. March, and D. J. Klein, J. Phys. Chem. A 111, 10162 (2007).

${ }^{57}$ H. Pedersen and L. Ojamäe, Nano Letters 6, 2004 (2006).

${ }^{58}$ H. B. Lal and K. Gaur, J. Mater. Sci. 23, 919 (1988). 
${ }^{59}$ B. Mercier, G. Ledoux, C. Dujardin, D. Nicolas, B. Masenelli, P. Melinon, and G. Bergeret, J. Chem. Phys. 126, 044507 (2007).

${ }^{60}$ B. Mercier, Ph.D. thesis, Universite Claude Bernard-Lyon I, 2005.

${ }^{61}$ F. Grosse, T. Watahiki, and W. Braun, Thin Solid Films 518, 4747 (2010).

${ }^{62}$ A. Prokofiev, A. Shelykh, and B. Melekh, J. Alloys Compd. 242, 41 (1996).

${ }^{63}$ F. X. Zhang, M. Lang, J. W. Wang, U. Becker, and R. C. Ewing, Phys. Rev. B 78, 064114 (2008).

${ }^{64}$ F. Cordero, F. Trequattrini, F. Deganello, V. La Parola, E. Roncari, and A. Sanson, Phys. Rev. B 82, 104102 (2010).

${ }^{65}$ B.-T. Melekh, V. Egorov, Y. Baikov, N. Kartenko, Y. Filin, M. Kompan, I. Novak, G. Venus, and V. Kulik, Solid State Ionics 97, 465 (1997).

${ }^{66}$ R. Hempelmann, Physica B 226, 72 (1996).
${ }^{67}$ Y. Yamazaki, C.-K. Yang, and S. M. Haile, Scr. Mater. 65, 102 (2011).

${ }^{68}$ D.-K. Lim, M.-B. Choi, K.-T. Lee, H.-S. Yoon, E. D. Wachsman, and S.-J. Song, Int. J. Hydrogen Energy 36, 9367 (2011).

${ }^{69}$ A. Grimaud, J.-M. Bassat, F. Mauvy, P. Simon, A. Canizares, B. Rousseau, M. Marrony, and J.-C. Grenier, Solid State Ionics 191, 24 (2011).

${ }^{70}$ S.-J. Song, E. D. Wachsman, S. E. Dorris, and U. Balachandran, Solid State Ionics 149, 1 (2002).

${ }^{71}$ D. R. Lick, CRC Handbook of Chemistry and Physics, 85th ed. (CRC Press, 2004-2005).

${ }^{72}$ S. S. Masterton, Chemical Principles (CBC College Publishing, 1983).

${ }^{73}$ L. V. Gurvich, Thermodynamic Properties of Individual Substances, Vols. 1-4 ( Nauka, Moskow, 1982-1987).

${ }^{74}$ N. I. Matskevich and T. A. Wolf, J. Chem. Thermodyn. 42, 225 (2010). 